\title{
TOTAL OVERLAPPING SCHWARZ' PRECONDITIONERS FOR ELLIPTIC PROBLEMS
}

\author{
Faker Ben Belgacem ${ }^{1}$, Nabil Gmati ${ }^{2}$ and Faten Jelassi $^{3,4}$
}

\begin{abstract}
A variant of the Total Overlapping Schwarz (TOS) method has been introduced in [Ben Belgacem et al., C. R. Acad. Sci., Sér. 1 Math. 336 (2003) 277-282] as an iterative algorithm to approximate the absorbing boundary condition, in unbounded domains. That same method turns to be an efficient tool to make numerical zooms in regions of a particular interest. The TOS method enjoys, then, the ability to compute small structures one wants to capture and the reliability to obtain the behavior of the solution at infinity, when handling exterior problems. The main aim of the paper is to use this modified Schwarz procedure as a preconditioner to Krylov subspaces methods so to accelerate the calculations. A detailed study concludes to a super-linear convergence of GMRES and enables us to state accurate estimates on the convergence speed. Afterward, some implementation hints are discussed. Analytical and numerical examples are also provided and commented that demonstrate the reliability of the TOS-preconditioner.
\end{abstract}

Mathematics Subject Classification. 65F08, 65N12, 65N80.

Received January 3rd, 2009. Revised September 12, 2009.

Published online April 15, 2010.

\section{INTRODUCTION}

Carrying relevant simulations of coupled models generally requires computational resources allowing to zoom on the regions of small scales. A significant part of the applications include fluid-particle flows such as blood flow (see [33]), structural mechanics with components having different (geometrical, physical) scales factors or fracture mechanics, cracks propagation and material fatigue (see [8]). Domain Decomposition methods are an affordable computational multi-scale methodology to handle those problems in a satisfactory way. Their use may be either as a tool to match multi-resolution approximations built on grids liable to embrace the local characteristics of the problem (see [5]) or as a powerful process to design performing sub-structuring preconditioners to solve the discrete system (see, e.g., $[32,36]$ ). Letting aside the most classical and popular domain decomposition approaches, recent attempts concentrate on the opportunity of coupling global computations with a zoom in the regions where small structures need to be simulated. To meet such requirements, some papers added appropriate improvements to existing computational methods. We especially refer to the Fat

\footnotetext{
Keywords and phrases. Total Overlapping Schwarz method, minimum residual Krylov methods, numerical zooms.

1 LMAC, Université de Technologie de Compiègne, BP 20529, 60205 Compiègne cedex, France.

${ }^{2}$ LAMSIN, École Nationale d'Ingénieurs de Tunis, B.P. 37, 1002 Le Belvédère, Tunisia. nabil.gmati@ipein.rnu.tn

3 LMAC, Université de Technologie de Compiègne, EA 2222, BP 20529, 60205 Compiègne cedex, France.

4 LAMSIN, Faculté des Sciences de Bizerte, Jarzouna, 7021 Bizerte, Tunisia.
} 
Boundary Method by Maury (see $[6,22,28]$ ), to the Numerical Zoom through the Schwarz Chimera method advocated by Pironneau's team (see $[2,7,21,31]$ ) and to the Multi-Scale Multi-Domain Method due the teams of Glowinski and Rappaz (see [14,15]). The modification of the Schwarz method introduced in [4] offers also the opportunity to make local computational zooms when and where needed. Though the 'upgraded' version is with Total Overlap its computational potentialities are substantially enhanced. The subproblems we cope with may be better conditioned and easier to solve accurately than those coming from the original version. The readers interested in further motivations may find instructive discussions in [15,21,28]. Fitting these methods into the Schwarz framework provides an appropriate variational tool to show their capabilities. Another highly attractive feature of the TOS method is the possibility to use it as a preconditioner to some Krylov subspaces methods to derive faster algorithms. We focus on the preconditioned GMRES algorithm, applied to problems set in cracked or perforated domains. Super-convergence results are therefore established. The same estimates are readily extended to more general Krylov algorithms such as the Bi-Conjugate Gradient method.

The contents of the paper are as follows. We consider a domain that contains cracks for which the TOS method is written. The singularities generated at the vicinity of the cracks might be numerically computed by means of local meshes with fine resolution or even using well adapted methods (think of the X-fem). Away from the cracks, the solution might be calculated by solving a problem on the safe domain (without cracks) which could be achieved by coarser grids and is therefore easier to solve. Both subproblems talk to each other through some appropriate transmission conditions which are the core of the new TOS method. They are exposed and commented in Section 1. Section 2 is devoted to the convergence analysis. The variational theory by Lions (see [24]) turns to be well-suited to the TOS method and results in a linear convergence rate. Afterwards, we make a static condensation of the problem in Section 3 which may be viewed as a TOS-preconditioning of the problem. We describe briefly, in Section 4, the GMRES algorithm applied to the pseudo-differential equation resulting from the static condensation. Section 5 supplies the proofs of its convergence. Tools currently used for the approximation of Kernel operators enable us to prove a super-linear convergence of the GMRES. In Section 6 , we apply the TOS method to perforated domains. We focus on the particular adaptation of the transmission conditions and briefly assess its efficiency. Section 7 consists in some hints to users that are possibly interested in implementing the TOS algorithm either as an independent solver or as a preconditioner to GMRES method. We close, in Section 8, by some analytical examples and we provide some numerical illustrations to support the theoretical analysis of this contribution. After the conclusion (Sect. 9) we provide in Section 10 the proof of some technical results required in the convergence study of GMRES method.

Some notations - Let $\Omega$ be a bounded domain in $\mathbb{R}^{d}, d=2,3$, with a Lipschitz boundary $\Gamma$. The generic points of $\Omega$ are denoted by $\boldsymbol{x}$ or $\boldsymbol{y}$. The Lebesgue space $L^{2}(\Omega)$ of square integrable complex valued functions is endowed with the natural norm $\|\cdot\|_{L^{2}(\Omega)}$ and we set $\boldsymbol{L}^{2}(\Omega)=L^{2}(\Omega)^{d}$. We need also some Sobolev spaces, $H^{1}(\Omega)$ involves all the functions that are in $L^{2}(\Omega)$ so as their partial derivatives. The subspace containing the functions in $H^{1}(\Omega)$ that vanish on $\Gamma$ is denoted by $H_{0}^{1}(\Omega)$. The set of the traces over $\Gamma$ of all the functions of $H^{1}(\Omega)$ is denoted $H^{1 / 2}(\Gamma)$ and $H^{-1 / 2}(\Gamma)$ is its dual (see [1]). The symbol [·] stands for the jump across a given boundary.

\section{Total OVERlapping SchWARZ AlgORIthm FOR CRACKS}

Let $\Omega$ be a bounded domain in $\mathbb{R}^{d}$ with a boundary $\Gamma$ being Lipschitz regular. We suppose that $\Omega$ is cracked along a connected Lipschitz curve $\gamma$, entirely embedded in $\Omega^{5}$. We denote $\Omega_{\gamma}=\Omega \backslash \gamma$ which is assumed connected. We exclude here any crack $\gamma$ that is a closed curve in $2 \mathrm{D}$ or a closed surface in $3 \mathrm{D}$. For these closed cracks, the problem can be uncoupled into an internal sub-problem, set in the sub-domain enclosed in $\gamma$, and in an external sub-problem, set in the perforated sub-domain. This latter configuration is fully considered in Section 6, dedicated to perforated domains.

\footnotetext{
${ }^{5}$ The assumption that $\gamma$ is connected does not restrict the generality.
} 

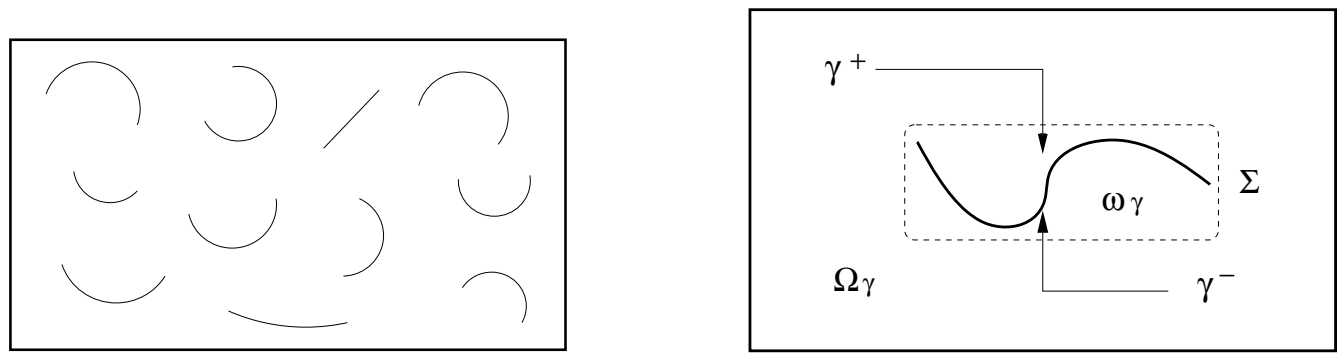

Figure 1. A multiply cracked domain to the left. A domain with a single crack to the right. The choice of $\Sigma$ is only indicative. A $\Sigma$ that embraces $\gamma$ is more judicious.

To specify the boundary conditions on the crack $\gamma$, we use the symbols $\gamma^{+}$and $\gamma^{-}$for both lips. We denote by $\boldsymbol{n}$ the unit normal to $\gamma$ oriented from $\gamma^{+}$toward $\gamma^{-}$(see Fig. 1). For a given data $f$ in $L^{2}\left(\Omega_{\gamma}\right)$, the Laplace problem consists in: finding $\varphi$ such that

$$
\begin{aligned}
& -\operatorname{div}(K \nabla \varphi)=f \quad \text { in } \Omega_{\gamma}, \\
& \varphi=0 \text { on } \Gamma \text {, } \\
& \left(K \partial_{\boldsymbol{n}}\right) \varphi=0 \quad \text { in } \gamma^{+} \cup \gamma^{-} \text {. }
\end{aligned}
$$

$K(\cdot)$ is a function in $L^{\infty}(\Omega)$ bounded away from zero ${ }^{6}$. Condition (1.3) says that $\gamma$ is a perfectly insulated crack while (1.2) is homogeneous only for simplification. The Sobolev space naturally attached to the Poisson problem (1.1)-(1.3) is given by

$$
H_{\Gamma}^{1}\left(\Omega_{\gamma}\right)=\left\{\psi \in L^{2}\left(\Omega_{\gamma}\right), \quad \nabla \psi \in \boldsymbol{L}^{2}\left(\Omega_{\gamma}\right), \quad \psi_{\mid \Gamma}=0\right\}
$$

Due to the connectedness of $\Omega_{\gamma}$, the semi-norm $|\cdot|_{H^{1}\left(\Omega_{\gamma}\right)}=\|\sqrt{K} \nabla \cdot\|_{L^{2}\left(\Omega_{\gamma}\right)}$ is actually a norm on $H_{\Gamma}^{1}\left(\Omega_{\gamma}\right)$ that is equivalent to $\|\cdot\|_{H^{1}\left(\Omega_{\gamma}\right)}($ see $[1])$.

Remark 1.1. Given that $\Omega_{\gamma}$ (the domain $\Omega$ minus the crack $\gamma$ ) is a (topological) open domain, the construction of the Sobolev space $H_{\Gamma}^{1}\left(\Omega_{\gamma}\right)$ follows the classical approach (see [17]), though, some comments may help understanding what happens at the vicinity of $\gamma$. First of all, notice that functions in $H_{\Gamma}^{1}\left(\Omega_{\gamma}\right)$ may have a jump across $\gamma$. We denote $[\psi]=\left(\psi_{\mid \gamma^{+}}-\psi_{\mid \gamma^{-}}\right)$that jump. [ $\left.\psi\right]$ belongs to $H_{00}^{1 / 2}(\gamma)$ (see [1] for the definition). This means that if $\tilde{\gamma}$ is a (Lipschitz) extension of $\gamma$, i.e. $\gamma \subset \subset \tilde{\gamma}^{7}$, and $[\psi]_{\mid \gamma}$ is extended by zero to $\tilde{\gamma}$, the resulting function belongs to $H^{1 / 2}(\tilde{\gamma})$. Actually, that extended function coincides exactly with $[\psi]_{\mid \tilde{\gamma}} \in H^{1 / 2}(\tilde{\gamma})$.

The variational formulation takes place in $H_{\Gamma}^{1}\left(\Omega_{\gamma}\right)$ and reads as: find $\varphi \in H_{\Gamma}^{1}\left(\Omega_{\gamma}\right)$ satisfying

$$
\int_{\Omega_{\gamma}} K \nabla \varphi \nabla \psi \mathrm{d} \boldsymbol{x}=\int_{\Omega_{\gamma}} f \psi \mathrm{d} \boldsymbol{x}, \quad \forall \psi \in H_{\Gamma}^{1}\left(\Omega_{\gamma}\right) .
$$

Now, let $\Sigma$ be a closed curve (or surface) in $\Omega_{\gamma}$ enclosing the crack $\gamma$ and denote by $\omega_{\gamma}$ the internal domain delimited by $\Sigma$ and containing $\gamma$. The multi-domain approach we pursue proceeds like the Schwarz algorithm with a particular arrangement in the transmission conditions that turns to be highly profitable. Two coupled subproblems replace (1.1)-(1.3). One subproblem, set on the whole domain $\Omega_{\gamma}$, is a transmission problem:

\footnotetext{
${ }^{6}$ The overall results described here remain valid for $K$, a symmetric positive definite tensor.

${ }^{7}$ We say that $\gamma$ is strongly embedded in $\tilde{\gamma}$.
} 
find $\eta \in H^{1}\left(\Omega_{\gamma}\right)$ such that

$$
\begin{aligned}
-\operatorname{div}(K \nabla \eta) & =f & & \text { in } \Omega_{\gamma} \\
{[\eta] } & =[\chi] & & \text { on } \gamma, \\
{\left[\left(K \partial_{n}\right) \eta\right] } & =0 & & \text { on } \gamma, \\
\eta & =0 & & \text { on } \Gamma .
\end{aligned}
$$

The other is set on $\omega_{\gamma}$ and is written as follows: find $\chi \in H^{1}\left(\omega_{\gamma}\right)$ such that

$$
\begin{aligned}
-\operatorname{div}(K \nabla \chi) & =f & & \text { in } \omega_{\gamma}, \\
\chi & =\eta & & \text { on } \Sigma \\
\left(K \partial_{n}\right) \chi & =0 & & \text { on } \gamma^{+} \cup \gamma^{-} .
\end{aligned}
$$

It can be seen that the difference $(\chi-\eta)$ is harmonic (with respect to div $(K \nabla(\cdot)))$ in $\omega_{\gamma}$, does not jump across $\gamma$ nor does its normal derivative and vanishes at $\Sigma$. We deduce thus that $\chi=\eta$ (in $\omega_{\gamma}$ ) and eventually $\chi=\varphi$ (in $\omega_{\gamma}$ ) and $\eta=\varphi\left(\right.$ in $\Omega_{\gamma}$ ). A similar approach appeared in [3] and is studied in [4,23] for exterior problems.

Remark 1.2. Let us emphasize on the fact that (1.5) and (1.6) are but transmission conditions. They are involved with the jumps $[\eta]$ and $\left[\left(K \partial_{\boldsymbol{n}}\right) \eta\right]$ and there is therefore no over-determination on $\gamma$. We refer to [11] for the analysis of similar problems.

Remark 1.3. The underlying idea of this decomposition is to apply a two-scales finite element method. A mesh of a small size is recommended in the local sub-domain $\omega_{\gamma}$ to capture the small scales at the vicinity of the crack (contained in $\chi$ ). Of a different resolution will be the mesh to compute $\eta$ in the global domain $\Omega$, which convey bigger scales far away from the cracks. The accuracy of the finite elements approximation of the coupled subproblems has been addressed successfully in [6] (see also [21] for a different multi-domain method).

The Total Overlapping Schwarz method consists in uncoupling both subproblems by computing iteratively two sequences. Assume $\left(\chi^{m}, \eta^{m}\right)$ is known, $\eta^{m+1} \in H_{\Gamma}^{1}\left(\Omega_{\gamma}\right)$ is the solution of

$$
\begin{aligned}
-\operatorname{div}\left(K \nabla \eta^{m+1}\right) & =f & & \text { in } \Omega_{\gamma}, \\
{\left[\eta^{m+1}\right] } & =\left[\chi^{m}\right] & & \text { on } \gamma, \\
{\left[\left(K \partial_{\boldsymbol{n}}\right) \eta^{m+1}\right] } & =0 & & \text { on } \gamma, \\
\eta^{m+1} & =0 & & \text { on } \Gamma,
\end{aligned}
$$

and $\chi^{m+1} \in H^{1}\left(\omega_{\gamma}\right)$ satisfies

$$
\begin{aligned}
-\operatorname{div}\left(K \nabla \chi^{m+1}\right) & =f & & \text { in } \omega_{\gamma}, \\
\chi^{m+1} & =\eta^{m+1} & & \text { on } \Sigma, \\
\left(K \partial_{n}\right) \chi^{m+1} & =0 & & \text { on } \gamma^{+} \cup \gamma^{-} .
\end{aligned}
$$

Obviously, the recurrence makes a mathematical sense, and results in two coherent sequences $\left(\chi^{m}\right)_{m} \subset H^{1}\left(\omega_{\gamma}\right)$ and $\left(\eta^{m}\right)_{m} \subset H_{\Gamma}^{1}\left(\Omega_{\gamma}\right)$.

The particularity of the TOS method consists in the boundary conditions inherited by $\eta^{m+1}$ from $\chi^{m}$ that are transmission conditions instead of the Dirichlet or Neumann conditions used in the standard version (see [32]). Given that $\omega_{\gamma}$ is entirely contained in $\Omega_{\gamma}$ (or simply in $\Omega$ ), this adapted Schwarz algorithm is with a total overlap and turns to be liable to make some zooming computations. The advantage is double. It is possible to make a zoom at the vicinity of the crack by employing specific discretizations such as X-fem, PU-fem or asymptotic expansion methods while avoiding the coupling with the finite elements approximation on the whole domain. 
The subproblem to cope with is therefore of a smaller size and may be reasonably solved with a fine resolution. Secondly, the condition number of the subproblem on $\eta^{m+1}$ does not suffer anymore from the crack. Indeed, when approximated by finite elements, the stiffness matrix is actually related to the safe domain $\Omega$ and the crack affects only the data part of the algebraic system. It is easier for users to employ efficient preconditioners for the safe domain than for the cracked or perforated one.

Remark 1.4. If convenient, one can entirely get rid of the crack $\gamma$ in problem (1.11)-(1.14), by considering any intermediary closed curve or surface $\gamma_{*}$ (between $\gamma$ and $\Sigma$ ). Indeed, we may consider the following problem: find $\tilde{\eta}^{m+1}$ such that

$$
\begin{aligned}
-\operatorname{div}\left(K \nabla \tilde{\eta}^{m+1}\right) & =\tilde{f} & & \text { in } \Omega \backslash \gamma_{*}, \\
{\left[\tilde{\eta}^{m+1}\right] } & =\chi^{m} & & \text { on } \gamma_{*}, \\
{\left[\left(K \partial_{\boldsymbol{n}}\right) \tilde{\eta}^{m+1}\right] } & =\left(K \partial_{\boldsymbol{n}}\right) \chi^{m} & & \text { on } \gamma_{*}, \\
\tilde{\eta}^{m+1} & =0 & & \text { on } \Gamma .
\end{aligned}
$$

$\tilde{f}$ is the extension of $f$ by zero in the sub-region of $\Omega$ inside $\gamma_{*}$. Following the proof [23], Lemma 2.1.3, it is readily checked that $\tilde{\eta}^{m+1}=\eta^{m+1}$ in the sub-domain outside $\gamma_{*}$. Hence, $\chi^{m+1}$ is not affected by the boundary condition $\chi^{m+1}=\tilde{\eta}^{m+1}$ on $\Sigma$. Notice that we have $\tilde{\eta}^{m+1}=\left(\eta^{m+1}-\chi^{m}\right)$ inside $\gamma_{*}$. Choosing $\gamma_{*}=\Sigma$ works as well, be only aware that the boundary condition $\chi=\tilde{\eta}$ in (1.9) comes from the external trace of $\tilde{\eta}^{m+1}$ on $\Sigma$. Notice that the introduction of such $\gamma_{*}$ to screen the cracks may have an interesting impact in the implementation when finite elements are used.

Remark 1.5. Things are even more advantageous for problems set on the whole space $\mathbb{R}^{d}$. Most often, the media is homogeneous at infinity so that $K$ may be fixed to one, and the operator $\operatorname{div}(K \nabla(\cdot))$ is a true Laplacian $\Delta(\cdot)$. The computation of $\eta^{m+1}$ is therefore explicit and very cheap which makes the modified Schwarz method even highly attractive for exterior problems (see $[3,23]$ ). Indeed, the problem on $\eta^{m+1}$ may be solved by integral representations. The Green function being given by $G(\boldsymbol{x}, \boldsymbol{y})=-\frac{1}{4 \pi} \frac{1}{|\boldsymbol{x}-\boldsymbol{y}|}$ in $\mathbb{R}^{3}$ and $G(\boldsymbol{x}, \boldsymbol{y})=-\frac{1}{2 \pi} \ln |\boldsymbol{x}-\boldsymbol{y}|$ in $\mathbb{R}^{2}$, we have a closed form for $\eta^{m+1}$,

$$
\eta^{m+1}(\boldsymbol{x})=\int_{\mathbb{R}^{d}} f(\boldsymbol{y}) G(\boldsymbol{x}, \boldsymbol{y}) \mathrm{d} \boldsymbol{x}-\int_{\gamma} \partial_{\boldsymbol{n}} G(\boldsymbol{x}, \boldsymbol{y})\left[\chi^{m}\right] \mathrm{d} \gamma .
$$

Notice that, in [3], we show how the promising adaptative process to approximate the exact absorbing boundary condition for the Maxwell system employed by Liu and Jin in [25] can be put under a Total Overlapping Schwarz form.

Remark 1.6. Let us emphasize on the fact that, in case the domain $\Omega_{\gamma}$ contains a finite number of connected cracks, the sub-domain $\omega_{\gamma}$ may be multiply connected. Each connected component encloses a single crack. Problem (1.15)-(1.17) is therefore composed of uncoupled local problems (one at the vicinity of each crack) that may be treated independently and in parallel computers. Notice also that the Schwarz algorithm can be written for emerging cracks and the analysis conducted following the same lines.

Remark 1.7. Let us consider a lifting operator $\mathcal{R}^{\Sigma}$ in $H^{1}(\omega)^{8}$ of traces on $\Sigma$, and $\breve{\eta} \in H^{1}(\Omega)$ be the solution of the Laplace equation in the safe domain

$$
\begin{aligned}
-\operatorname{div}(K \nabla \breve{\eta})=f & & \text { in } \Omega, \\
\breve{\eta}=0 & & \text { on } \Gamma .
\end{aligned}
$$

\footnotetext{
${ }^{8} \omega$ is the safe sub-domain.
} 
Starting from the splitting $(\eta, \chi)=\left(\underline{\eta}+\breve{\eta}, \underline{\chi}+\mathcal{R}^{\Sigma}(\eta)\right)$ it may be straightforwardly checked that $\underline{\chi} \in H_{\Sigma}^{1}\left(\omega_{\gamma}\right)$ is the solution of the variational problem

$$
\int_{\omega_{\gamma}} K \nabla \underline{\chi} \nabla \psi \mathrm{d} \boldsymbol{x}+\int_{\omega_{\gamma}} K \nabla \mathcal{R}^{\Sigma}(\underline{\eta}) \nabla \psi \mathrm{d} \boldsymbol{x}=\int_{\omega_{\gamma}} f \psi \mathrm{d} \boldsymbol{x}-\int_{\omega_{\gamma}} K \nabla \mathcal{R}^{\Sigma}(\breve{\eta}) \nabla \psi \mathrm{d} \boldsymbol{x}, \quad \forall \psi \in H_{\Sigma}^{1}\left(\omega_{\gamma}\right) .
$$

This equation may be re-transcribed in a compact form as follows

$$
\mathcal{A} \underline{\underline{\chi}}+\mathcal{C} \underline{\eta}^{\Sigma}=F-\mathcal{C} \breve{\eta}^{\Sigma}, \quad \text { in }\left(H_{\Sigma}^{1}\left(\omega_{\gamma}\right)\right)^{\prime}
$$

where $\underline{\eta}^{\Sigma}=\underline{\eta}_{\mid \Sigma}$. The operator $\mathcal{A}$ is the elliptic operator $(-\operatorname{div}(K \nabla \cdot))$ in $\omega_{\gamma}$ with a Dirichlet condition on $\Sigma$ and a Neumann condition on $\gamma$. It is invertible. Observing, now, that $\underline{\eta}$ depends linearly on $\underline{\chi}\left(\right.$ actually on $\left.[\underline{\chi}]_{\mid \gamma}\right)$, we derive the final equation on $\underline{\chi} \in H_{\Sigma}^{1}\left(\omega_{\gamma}\right)$,

$$
(\mathcal{A}+\mathcal{C} \mathcal{T}) \underline{\chi}=F-\mathcal{C} \breve{\eta}^{\Sigma}, \quad \text { in }\left(H_{\Sigma}^{1}\left(\omega_{\gamma}\right)\right)^{\prime}
$$

The composed operator $(\mathcal{C T})$ ensures the coupling between $[\underline{\chi}]_{\mid \gamma}$ and $\eta_{\mid \Sigma}$. The same arguments applied to the sequences $\left(\chi^{m}, \eta^{m}\right)$ show that $\left(\underline{\chi}^{m}\right)_{m}$ obeys

$$
\underline{\chi}^{m+1}+(\mathcal{A})^{-1}(\mathcal{C} \mathcal{T}) \underline{\chi}^{m}=(\mathcal{A})^{-1}\left(F-\mathcal{C} \breve{\eta}^{\Sigma}\right), \quad \text { in } H_{\Sigma}^{1}\left(\omega_{\gamma}\right)
$$

Then, the TOS method turns to be the preconditioned Richardson algorithm applied to $(1.21)^{9}$. $\mathcal{A}$ is the preconditioner called henceforth the TOS-preconditioner. Problem (1.22) is hence the TOS-preconditioned form of (1.21). Back to $(\chi, \eta)$, we obtain easily that $\left(\eta^{m+1}, \chi^{m+1}\right)=\left(\underline{\eta}^{m+1}+\breve{\eta}, \underline{\chi}^{m+1}+\mathcal{R}^{\Sigma}\left(\eta^{m+1}\right)\right)$.

\section{Convergence of the total overlapping Schwarz method}

The convergence of the TOS method is the aim here. A variational approach similar to [24] is briefly discussed. Consider the error $e^{m}=\left(\chi-\chi^{m}\right)$ and $\tau^{m}=\left(\eta-\eta^{m}\right)$. Subtracting the exact problems (1.8)-(1.10) on $\chi$ and (1.4)-(1.7) on $\eta$ from their Schwarz counterparts (1.15)-(1.17) and (1.11)-(1.14) yields that $e^{m}$ and $\tau^{m}$ are related to each other through the same partial differential equations (1.15)-(1.17) and (1.11)-(1.14) where $f=0$. The convergence analysis is conducted in the space $H_{\Gamma}^{1}\left(\Omega_{\gamma}\right)$. We need, therefore, to extend $e^{m+1} \in H^{1}\left(\omega_{\gamma}\right)$ by $\tau^{m+1}$, in $\Omega_{\gamma} \backslash \omega_{\gamma}$. The resulting function has no jump across $\Sigma$ and, hence, belongs to $H_{\Gamma}^{1}\left(\Omega_{\gamma}\right)$. By a notation abuse we still denote by $e^{m+1}$ this extended function.

Using equations (1.11)-(1.14) on $\tau^{m+1}$, we deduce the variational relation (we set $V_{1}=H_{0}^{1}(\Omega)$ )

$$
\int_{\Omega_{\gamma}} K \nabla \tau^{m+1} \nabla \psi \mathrm{d} \boldsymbol{x}=0, \quad \forall \psi \in V_{1}
$$

Plugging $e^{m}$, it comes out that

$$
\int_{\Omega_{\gamma}} K \nabla\left(\tau^{m+1}-e^{m}\right) \nabla \psi \mathrm{d} \boldsymbol{x}=\int_{\Omega_{\gamma}} K \nabla\left(-e^{m}\right) \nabla \psi \mathrm{d} \boldsymbol{x}, \quad \forall \psi \in V_{1} .
$$

Since $\left[\tau^{m+1}-e^{m}\right]_{\mid \gamma}=0$, then $\left(\tau^{m+1}-e^{m}\right)$ belongs to $V_{1}$ and we have that

$$
\tau^{m+1}=\left(I-P_{1}\right)\left(e^{m}\right),
$$

\footnotetext{
${ }^{9}$ It is also a Jacobi type algorithm.
} 
where $P_{1}$ is the orthogonal projection on $V_{1}$ with respect to the semi-norm $|\cdot|_{H^{1}(\Omega)}$. Next, so as to get a similar identity we introduce the closed subspace $V_{2}$ of $H_{\Gamma}^{1}\left(\Omega_{\gamma}\right)$ defined by

$$
V_{2}=\left\{\psi \in H_{\Gamma}^{1}\left(\Omega_{\gamma}\right), \quad(\psi)_{\mid\left(\Omega_{\gamma} \backslash \omega_{\gamma}\right)}=0\right\} .
$$

$V_{2}$ can be identified with $H_{\Sigma}^{1}\left(\omega_{\gamma}\right)$. The subproblem (1.15)-(1.17) (with $f=0$ ) on $e^{m+1}$ can be rewritten under the variational form

$$
\int_{\omega_{\gamma}} K \nabla\left(e^{m+1}\right) \nabla \psi \mathrm{d} \boldsymbol{x}=0, \quad \forall \psi \in V_{2},
$$

which can be reformulated as follows

$$
\int_{\Omega_{\gamma}} K \nabla\left(e^{m+1}-\tau^{m+1}\right) \nabla \psi \mathrm{d} \boldsymbol{x}=\int_{\Omega_{\gamma}} K \nabla\left(-\tau^{m+1}\right) \nabla \psi \mathrm{d} \boldsymbol{x}, \quad \forall \psi \in V_{2} .
$$

Clearly $\left(e^{m+1}-\tau^{m+1}\right)$ lies in $V_{2}$. As a result, we obtain that

$$
e^{m+1}=\left(I-P_{2}\right)\left(\tau^{m+1}\right),
$$

$P_{2}$ being the orthogonal projection on $V_{2}$ with respect to $|\cdot|_{H^{1}\left(\Omega_{\gamma}\right)}$. Putting together (2.1) and (2.2) yields the inductions

$$
\begin{aligned}
\tau^{m+1} & =\left(I-P_{1}\right)\left(I-P_{2}\right)\left(\tau^{m}\right), \\
e^{m+1} & =\left(I-P_{2}\right)\left(I-P_{1}\right)\left(e^{m}\right) .
\end{aligned}
$$

Both formulas allow to state a linear convergence result.

Proposition 2.1. The TOS method converges with a linear rate, that is, there exists $\zeta \in[0,1[$ such that

$$
\left|\varphi-\chi^{m}\right|_{H^{1}\left(\omega_{\gamma}\right)}+\left|\varphi-\eta^{m}\right|_{H^{1}\left(\Omega_{\gamma}\right)} \leq C\left(\chi^{0}\right) \zeta^{m}, \quad \forall m \geq 0 .
$$

Proof. We have to state that the errors $\left|\tau^{m}\right|_{H^{1}\left(\Omega_{\gamma}\right)}$ and $\left|e^{m}\right|_{H^{1}\left(\Omega_{\gamma}\right)}$ decay towards zero. This is achieved if the operators $\left(I-P_{1}\right)$ and $\left(I-P_{2}\right)$ are proven to be contractions which can be proceeded like in Lions in [24]. It is sufficient to remark that the following decomposition holds

$$
H_{\Gamma}^{1}\left(\Omega_{\gamma}\right)=V_{1}+V_{2},
$$

which is straightforward. The proof is complete.

\section{Static CONDENSATion}

We aim a static condensation of the problem on the boundary $\Sigma$. Let us therefore define $\check{\chi} \in H^{1}\left(\omega_{\gamma}\right)$ to be the solution of

$$
\begin{aligned}
& -\operatorname{div}(K \nabla \check{\chi})=f \quad \text { in } \omega_{\gamma}, \\
& \check{\chi}=0 \text { on } \Sigma \text {, } \\
& \left(K \partial_{n}\right) \check{\chi}=0 \quad \text { on } \gamma^{+} \cup \gamma^{-} \text {, }
\end{aligned}
$$


and $\check{\eta} \in H^{1}\left(\Omega_{\gamma}\right)$ that satisfies

$$
\begin{aligned}
-\operatorname{div}(K \nabla \check{\eta}) & =f & & \text { in } \Omega_{\gamma}, \\
{[\check{\eta}] } & =[\check{\chi}] & & \text { on } \gamma, \\
{\left[\left(K \partial_{\boldsymbol{n}}\right) \check{\eta}\right] } & =0 & & \text { on } \gamma, \\
\check{\eta} & =0 & & \text { on } \Gamma .
\end{aligned}
$$

Notice that there is no 'real coupling' between both problems. We solve the first and all the data necessary for the second are available to come up with $\check{\eta}$. Let $\lambda \in H^{1 / 2}(\Sigma)$ and $\chi_{\lambda} \in H^{1}\left(\omega_{\gamma}\right)$ be the solution of

$$
\begin{aligned}
& -\operatorname{div}\left(K \nabla \chi_{\lambda}\right)=0 \quad \text { in } \omega_{\gamma}, \\
& \chi_{\lambda}=\lambda \text { on } \Sigma, \\
& \left(K \partial_{n}\right) \chi_{\lambda}=0 \quad \text { on } \gamma^{+} \cup \gamma^{-} \text {, }
\end{aligned}
$$

and $\eta_{\lambda} \in H^{1}(\Omega)$ such that

$$
\begin{aligned}
-\operatorname{div}\left(K \nabla \eta_{\lambda}\right) & =0 & & \text { in } \Omega_{\gamma}, \\
{\left[\eta_{\lambda}\right] } & =\left[\chi_{\lambda}\right] & & \text { on } \gamma, \\
{\left[\left(K \partial_{\boldsymbol{n}}\right) \eta_{\lambda}\right] } & =0 & & \text { on } \gamma, \\
\eta_{\lambda} & =0 & & \text { on } \Gamma .
\end{aligned}
$$

It can be checked that the solution $(\chi, \eta)$ can be reconstructed through the decomposition $(\check{\chi}, \check{\eta})+\left(\chi_{\lambda}, \eta_{\lambda}\right)$ provided that $\lambda \in H^{1 / 2}(\Sigma)$ solves the equation

$$
\left(\mathcal{I}+\mathcal{B}_{\Sigma}\right)(\lambda)=F, \quad \text { in } H^{1 / 2}(\Sigma)
$$

In $(3.1), \mathcal{I}$ is the identity and we have set

$$
\mathcal{B}_{\Sigma}(\lambda)=-\left(\eta_{\lambda}\right)_{\mid \Sigma}, \quad F=(\check{\eta})_{\mid \Sigma}
$$

The linear operator $\mathcal{B}_{\Sigma}$ mapping $H^{1 / 2}(\Sigma)$ into $H^{1 / 2}(\Sigma)$ is compact thanks to the elliptic theory for the problem on $\eta_{\lambda}$ (see [17]). Indeed, we have that $\mathcal{B}_{\Sigma}(\lambda)$ belongs to $H^{1 / 2+\epsilon}(\Sigma), \epsilon>0^{10}$ with the stability

$$
\left\|\mathcal{B}_{\Sigma}(\lambda)\right\|_{H^{1 / 2+\epsilon(\Sigma)}} \leq C\|\lambda\|_{H^{1 / 2}(\Sigma)} .
$$

Moreover, Lemma 10.1 states that $\left(\mathcal{I}+\mathcal{B}_{\Sigma}\right)$ is an isomorphism. As a result, equation (3.1) is well posed and has only one solution. Using Richardson algorithm yields the induction

$$
\lambda^{m+1}+\mathcal{B}_{\Sigma}\left(\lambda^{m}\right)=F .
$$

One can verify that this is but another way to express the iterative algorithm where $\left(\chi^{m}, \eta^{m}\right)=\left(\chi_{\lambda^{m}}, \eta_{\lambda^{m}}\right)+$ $(\check{\chi}, \check{\eta})$. Because (3.3) is equivalent to (the TOS-preconditioned) equation (1.22), it is but another form of the TOS-preconditioned equation. Accelerating the convergence can be obtained by some Residual Krylov subspaces methods applied to the linear problem (3.1). These methods consist in minimizing the norm of the residual on some Krylov subspaces. Handling it in the Lebesgue space $L^{2}(\Sigma)$ instead of the dual space $H^{1 / 2}(\Sigma)$ brings more flexibility in the computations as well as in the theoretical analysis even though the same study can be carried out in $H^{1 / 2}(\Sigma)$ following the same arguments. Notice that $\mathcal{B}_{\Sigma}$ is a compact operator on $L^{2}(\Sigma)$ and that by Lemma $10.2,\left(\mathcal{I}+\mathcal{B}_{\Sigma}\right)$ is an isomorphism on $L^{2}(\Sigma)$.

\footnotetext{
${ }^{10}$ This depends on the geometry of the fictitious boundary $\Sigma$. Let us emphasize on that the user's choice of $\Sigma$ is totally free.
} 


\section{KRYLOV SUBSPACES MEthods}

Minimum Residual Iterative methods consist of a sequence of least squares problems set in Krylov subspaces, the cost function to be minimized is the $\left(L^{2}(\Sigma)-\right)$ norm of the residual. The most popular of these methods is GMRES (see [34]). The extension of it to infinite dimensional spaces has been successfully realized in [29].

We denote, for convenience, by $\mathcal{H}_{\Sigma}=\left(\mathcal{I}+\mathcal{B}_{\Sigma}\right)$ and define the Krylov subspaces of order $m$,

$$
K_{m}\left(\mathcal{H}_{\Sigma}, F\right)=\operatorname{SPAN}\left\{F, \mathcal{H}_{\Sigma} F,\left(\mathcal{H}_{\Sigma}\right)^{2} F, \ldots,\left(\mathcal{H}_{\Sigma}\right)^{m-1} F\right\}
$$

GMRES applied to (3.1) with the initial guess $\lambda_{0}=0$ computes iterates that are solutions of the minimization

$$
\left\|\left(\mathcal{H}_{\Sigma}\right) \lambda_{m}-F\right\|_{L^{2}(\Sigma)}=\min _{\mu \in K_{m}\left(\mathcal{H}_{\Sigma}, F\right)}\left\|\left(\mathcal{H}_{\Sigma}\right) \mu-F\right\|_{L^{2}(\Sigma)}
$$

The Krylov subspaces form an increasing sequence, $K_{m}\left(\mathcal{H}_{\Sigma}, F\right) \subset K_{m+1}\left(\mathcal{H}_{\Sigma}, F\right)$. The GMRES method may break down when the equality occurs, say at iteration $m^{*}$ for the first time. Thus the exact solution $\lambda$ belongs to $K_{m^{*}}\left(\mathcal{H}_{\Sigma}, F\right)$ and the algorithm converges after exactly $m^{*}$ iterations. Otherwise, let us denote by $r_{m}=$ $\left(\mathcal{H}_{\Sigma} \lambda_{m}-F\right)$ the residual at the iteration $m$ and $\mathcal{P}_{m}$ be the space of polynomials with degree $\leq m$. We have that (see [16])

$$
\left\|r_{m}\right\|_{L^{2}(\Sigma)}=\min _{q \in \mathcal{P}_{m}, q(0)=1}\left\|q\left(\mathcal{H}_{\Sigma}\right) F\right\|_{L^{2}(\Sigma)}=\min _{q \in \mathcal{P}_{m}, q(1)=1}\left\|q\left(\mathcal{B}_{\Sigma}\right) F\right\|_{L^{2}(\Sigma)}
$$

Given that the operator $\mathcal{B}_{\Sigma}$ is compact, following Moret, GMRES method converges (see [29]). We are interested in a further knowledge of the convergence rate of the algorithm which depends on the properties of the spectrum of $\mathcal{B}_{\Sigma}$. Let $\left(\mu_{p}\right)_{p \geq 0}$ be the singular values of the compact operator $\mathcal{B}_{\Sigma}$, and $\left(\nu_{p}\right)_{p \geq 0}$ are the singular values of $\left(\mathcal{H}_{\Sigma}\right)^{-1}$ both sorted in a non increasing order. The sequence $\left(\mu_{p}\right)_{p \geq 0}$ decays toward zero while $\left(\nu_{p}\right)_{p \geq 0}$ is bounded and bounded away from zero since, $\mathcal{H}_{\Sigma}$ is an isomorphism on $L^{2}(\Sigma)$.

Lemma 4.1 ([29], Thm. 1). The following bound holds

$$
\left\|r_{m}\right\|_{L^{2}(\Sigma)} \leq\left(\prod_{p=0}^{m-1} \mu_{p} \nu_{p}\right)\|F\|_{L^{2}(\Sigma)} .
$$

The abstract estimate (4.3) tells that the convergence speed of GMRES is driven by the singular values of $\mathcal{B}_{\Sigma}$. Accordingly, our main objective in the sequel is the behavior of those singular values. Thanks to the Weyl theory, this issue is related to the compactness degree of the operator $\mathcal{B}_{\Sigma}$ (see [37]). That is why we put $\mathcal{B}_{\Sigma}$ under an integral form. The kernel related to $\mathcal{B}_{\Sigma}$ can be obtained by using $G(\cdot, \cdot)$, the Green function of the elliptic problem in the safe domain $\Omega$. Let $\boldsymbol{x} \in \Omega$, then $G(\boldsymbol{x}, \cdot)$ is the solution of

$$
\begin{aligned}
-\operatorname{div}(K \nabla G)(\boldsymbol{x}, \cdot) & =\delta_{\boldsymbol{x}} & & \text { in } \Omega, \\
G(\boldsymbol{x}, \cdot) & =0 & & \text { on } \Gamma .
\end{aligned}
$$

$\delta_{\boldsymbol{x}}$ is the Dirac distribution centered at $\boldsymbol{x}$. The function $G(\cdot, \cdot)$ belongs to $L^{2}(\Omega \times \Omega)$, is symmetric and, by the De Giorgio-Nash theorem, is continuous everywhere in $\Omega \times \Omega$ excepted on the diagonal part of it (see [11]). Consequently, using an integration by parts we derive that

$$
\left(\mathcal{B}_{\Sigma} \lambda\right)(\boldsymbol{x})=-\eta_{\lambda}(\boldsymbol{x})=\int_{\gamma}\left(K \partial_{\boldsymbol{n}_{\boldsymbol{y}}}\right) G(\boldsymbol{x}, \boldsymbol{y})\left[\chi_{\lambda}\right](\boldsymbol{y}) \mathrm{d} \gamma_{\boldsymbol{y}}, \quad \forall \boldsymbol{x} \in \Sigma
$$

The symbol $\boldsymbol{n}_{\boldsymbol{y}}$ is used for $\left(\boldsymbol{n}(\boldsymbol{y}) \cdot \nabla_{\boldsymbol{y}}\right)$. 


\section{Convergence of the GMres}

To investigate the singular values of $\mathcal{B}_{\Sigma}$ we need first to characterize $\left(\mu_{p}\right)_{p \geq 0}$ by the Courant-Weyl min-max principle (see $[29,37])$

$$
\mu_{p}=\min _{\left(\mathcal{B}_{\Sigma}\right)_{p}}\left\|\mathcal{B}_{\Sigma}-\left(\mathcal{B}_{\Sigma}\right)_{p}\right\|_{\mathcal{L}\left(L^{2}(\Sigma)\right)}
$$

where $\left(\mathcal{B}_{\Sigma}\right)_{p \geq 0}$ runs through the set of the linear operators in $L^{2}(\Sigma)$ with rank $\leq p$. The result we pursue is that the singular values of $\mathcal{B}_{\Sigma}$ decay exponentially fast. Asymptotics for the singular values of integral operators may be related of the smoothness of their kernels (see [37]). The construction of a $\left(\mathcal{B}_{\Sigma}\right)_{p}$ close to $\left(\mathcal{B}_{\Sigma}\right)$ relies on the accurate approximability of the kernel of the operator $\mathcal{B}_{\Sigma}{ }^{11}$ by some Fourier expansions on $\Sigma$. Recall here again that the choice of $\Sigma$ is left to users who may fix it to a piecewise analytic curve (or surface) located in the regions of regularity (constancy!) of $K(\cdot)$ so as to ensure the best possible regularity of $\boldsymbol{x} \mapsto G(\cdot, \boldsymbol{y}), \boldsymbol{y} \in \gamma$. We expose the proof for the Laplace operator $(K=1)$ for which the regularity of the Green kernel $G(\cdot, \cdot)$ at the vicinity of $\Sigma \times \gamma$ is fully elucidated (see $[12,18]$ ). We draw the reader's attention to the fact that for general $K(\cdot)$, we may proceed following the same arguments provided that the smoothness of the associated Green function $G(\cdot, \cdot)$ is available. This is expected to be only connected to the smoothness of $K(\cdot)$ at the vicinity of $\Sigma$. To our knowledge such results are still missing. This is the reason why we focus on the particular Poisson equation. Actually, for this case we provide an improved proof to show that the exponential decreasing rate for the singular-values depends also on the distance $d(\Sigma, \gamma)$. We will consider separately the cases $d=2$ and $d=3$.

Proposition 5.1. Let $d=2$ and $K(\cdot)=1$. Assume that the fictitious boundary $\Sigma$ is Lipschitz regular. It holds that

$b$ is a positive constant and $\zeta \in] 0,1[$.

$$
\mu_{p} \leq b \zeta^{p}, \quad \forall p \geq 0
$$

Proof. The proof is structured into three steps. The first is dedicated to the transformation of the geometry and the two others for the core of the proof.

(i) Because they do not intersect the closed curves (or surfaces) $\gamma$ and $\Sigma$ can be separated by a closed Jordan curve $\Pi$ which splits $\Omega$ into two connected regions, a bounded domain $\Omega_{\Pi}$ that encloses the crack and an exterior domain $\Omega_{\Pi}^{\prime}$ that contains $\Sigma$. Then, $\Omega_{\Pi}^{\prime}$ is transformed by means of a conformal map into the annular domain $\left(\Omega_{\Pi}^{\prime}\right)_{*}$ with double radius $(\varrho, R)$ (see [35]). The curve $\Pi$ is mapped into the circle of radius $\varrho$ denoted $\Pi_{*}$, $\Gamma$ into the circle of radius $R$ denoted $\Gamma_{*}$ and $\Sigma$ into a $\Sigma_{*}$ which is not necessarily a circle. Next, changing the variable $\boldsymbol{x} \in \Sigma$ into $\boldsymbol{x}_{*} \in \Sigma_{*}$ in (4.6) we obtain a new Kernel operator $\mathcal{B}_{\Sigma_{*}}$ where $\boldsymbol{H}(\cdot, \cdot)=\nabla_{\boldsymbol{y}} G(\cdot, \cdot)$ is replaced by $\boldsymbol{H}_{*}(\cdot, \cdot)=\nabla_{\boldsymbol{y}} G_{*}(\cdot, \cdot)$. Notice that once these transformations achieved, we are no longer allowed to view $\left(\Omega_{\Pi}^{\prime}\right)_{*}$ as a continuation of the domain enclosed by $\Pi$ (the one that contains the crack). Both domains should be considered as sets located in different spaces. The caution to observe strictly during the proof is therefore the impossibility to interchange $\boldsymbol{x}_{*}$ and $\boldsymbol{y}$.

(ii) That $\gamma$ and $\Omega_{\Pi}^{\prime}$ are distant from each other $\left(d\left(\gamma, \Omega_{\Pi}^{\prime}\right)>0\right)$ makes the kernel $\boldsymbol{H}(\cdot, \cdot)$ be uniformly bounded $($ see $[12,18])$

$$
|\boldsymbol{H}(\boldsymbol{x}, \boldsymbol{y})| \leq M, \quad \forall(\boldsymbol{x}, \boldsymbol{y}) \in \Omega_{\Pi}^{\prime} \times \gamma .
$$

$M>0$ is a given constant that increases when the distant $d\left(\gamma, \Omega_{\Pi}^{\prime}\right)$ decreases. The changing of the variable $\boldsymbol{x} \mapsto \boldsymbol{x}_{*}$ preserves this estimate for $\boldsymbol{H}_{*}(\cdot, \cdot)$. It reads as follows

$$
\left|\boldsymbol{H}_{*}\left(\boldsymbol{x}_{*}, \boldsymbol{y}\right)\right| \leq M, \quad \forall\left(\boldsymbol{x}_{*}, \boldsymbol{y}\right) \in\left(\Omega_{\Pi}^{\prime}\right)_{*} \times \gamma
$$

To alleviate the presentation, the $\operatorname{symbol}_{*}$ is removed in the notations from now on. For instance, $H(\boldsymbol{x}, \boldsymbol{y})$ is hence employed instead of $H_{*}\left(\boldsymbol{x}_{*}, \boldsymbol{y}\right)$ and $\Sigma$ is used for $\Sigma_{*}$. We identify $\boldsymbol{x} \in \Omega_{\Pi}^{\prime}$ (in reality $\boldsymbol{x}_{*} \in\left(\Omega_{\Pi}^{\prime}\right)_{*}$ )

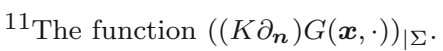


with its complex affix we still denote abusively by $\boldsymbol{x}$ in some places and by $r \mathrm{e}^{\mathrm{i} \theta}$ in other places, where $(r, \theta) \in[\varrho, \infty[\times[0,2 \pi[$ are the polar coordinates.

(iii) Conformal mappings preserve the harmonicity. Hence, for any $\boldsymbol{y} \in \gamma$, the function $\boldsymbol{H}(\cdot, \boldsymbol{y})$ is harmonic in $\Omega_{\Pi}^{\prime}$. A variable separation in the polar referential $(r, \theta)$, leads to the expansion $\left(\boldsymbol{x}=r \mathrm{e}^{\mathrm{i} \theta},(r, \theta) \in[\varrho, R] \times\right.$ $[0,2 \pi])$,

$$
\boldsymbol{H}\left(r \mathrm{e}^{\mathrm{i} \theta}, \boldsymbol{y}\right)=\left(\boldsymbol{a}_{0}(\boldsymbol{y})+\boldsymbol{b}_{0}(\boldsymbol{y}) \ln r\right)+\sum_{k=1}^{\infty}\left(\boldsymbol{a}_{k}(\boldsymbol{y}) r^{k}+\boldsymbol{b}_{k}(\boldsymbol{y}) r^{-k}\right) \mathrm{e}^{\mathrm{i} k \theta} .
$$

The cœfficients $\boldsymbol{a}_{k}, \boldsymbol{b}_{k} \in \mathbb{R}^{2}$, for $k \geq 1$, are specified by means of the following integrals

$$
\begin{aligned}
& \boldsymbol{a}_{k}(\boldsymbol{y})=\frac{-1}{2 \pi\left(R^{k} \varrho^{-k}-R^{-k} \varrho^{k}\right)}\left(R^{-k} \int_{0}^{2 \pi} \boldsymbol{H}\left(\varrho \mathrm{e}^{\mathrm{i} \theta}, \boldsymbol{y}\right) \mathrm{e}^{-\mathrm{i} k \theta} \mathrm{d} \theta-\varrho^{-k} \int_{0}^{2 \pi} \boldsymbol{H}\left(R \mathrm{e}^{\mathrm{i} \theta}, \boldsymbol{y}\right) \mathrm{e}^{-\mathrm{i} k \theta} \mathrm{d} \theta\right), \\
& \boldsymbol{b}_{k}(\boldsymbol{y})=\frac{1}{2 \pi\left(R^{k} \varrho^{-k}-R^{-k} \varrho^{k}\right)}\left(R^{k} \int_{0}^{2 \pi} \boldsymbol{H}\left(\varrho \mathrm{e}^{\mathrm{i} \theta}, \boldsymbol{y}\right) \mathrm{e}^{-\mathrm{i} k \theta} \mathrm{d} \theta-\varrho^{k} \int_{0}^{2 \pi} \boldsymbol{H}\left(R \mathrm{e}^{\mathrm{i} \theta}, \boldsymbol{y}\right) \mathrm{e}^{-\mathrm{i} k \theta} \mathrm{d} \theta\right) .
\end{aligned}
$$

Owing to the boundedness of $\boldsymbol{H}(\cdot, \cdot)$ on $\Omega_{\Pi}^{\prime} \times \gamma$ in (5.2), the following estimates can be stated for $\left(\boldsymbol{a}_{k}, \boldsymbol{b}_{k}\right)_{k \geq 1}$,

$$
\max _{\boldsymbol{y} \in \gamma}\left|\boldsymbol{a}_{k}(\boldsymbol{y})\right| \leq M^{\prime} R^{-k}, \quad \max _{\boldsymbol{y} \in \gamma}\left|\boldsymbol{b}_{k}(\boldsymbol{y})\right| \leq M^{\prime} \varrho^{k} .
$$

Let now $p$ be a given odd integer and denote $p^{*}$ the integer part of $p / 2$. We consider the kernel determined by the partial sum

$$
\boldsymbol{H}_{p}\left(r \mathrm{e}^{\mathrm{i} \theta}, \boldsymbol{y}\right)=\left(\boldsymbol{a}_{0}(\boldsymbol{y})+\boldsymbol{b}_{0}(\boldsymbol{y}) \ln r\right)+\sum_{k=1}^{p^{*}}\left(\boldsymbol{a}_{k}(\boldsymbol{y}) r^{k}+\boldsymbol{b}_{k}(\boldsymbol{y}) r^{-k}\right) \mathrm{e}^{\mathrm{i} k \theta} .
$$

Bounding $\left(\boldsymbol{H}-\boldsymbol{H}_{p}\right)(\cdot, \boldsymbol{y})$ on $\Sigma$ is realized as follows $\left(\boldsymbol{x}=r \mathrm{e}^{\mathrm{i} \theta} \in \Sigma\right)$

$$
\left|\boldsymbol{H}\left(r \mathrm{e}^{\mathrm{i} \theta}, \boldsymbol{y}\right)-\boldsymbol{H}_{p}\left(r \mathrm{e}^{\mathrm{i} \theta}, \boldsymbol{y}\right)\right| \leq \sum_{k>p^{*}}\left|\boldsymbol{a}_{k}(\boldsymbol{y})\right| r^{k}+\sum_{k>p^{*}}\left|\boldsymbol{b}_{k}(\boldsymbol{y})\right| r^{-k}, \quad \forall \boldsymbol{y} \in \gamma .
$$

Using (5.4), we obtain a uniform estimate

$$
\max _{\boldsymbol{y} \in \gamma}\left|\boldsymbol{H}(\boldsymbol{x}, \boldsymbol{y})-\boldsymbol{H}_{p}(\boldsymbol{x}, \boldsymbol{y})\right| \leq M^{\prime} \sum_{k>p^{*}}\left(\frac{r}{R}\right)^{k}+M^{\prime} \sum_{k>p^{*}}\left(\frac{r}{\varrho}\right)^{-k} .
$$

Noticing that $\Sigma$ is a compact curve enclosing $\Pi$ (the circle with radius $\varrho$ ) and encircled by $\Gamma$ (the circle with radius $R$ ). Since they have no common part the new parameter

$$
\zeta=\left(\max _{r=|\boldsymbol{x}|, \boldsymbol{x} \in \Sigma} \max \left(\frac{\varrho}{r}, \frac{r}{R}\right)\right)^{1 / 2}=\left(\max _{\boldsymbol{x} \in \Sigma} \max \left(\frac{\varrho}{|\boldsymbol{x}|}, \frac{|\boldsymbol{x}|}{R}\right)\right)^{1 / 2}
$$

is necessarily smaller than 1 . Back to the error on $\boldsymbol{H}(\cdot, \cdot)$ we derive a full uniform bound ${ }^{12}$

$$
\max _{(\boldsymbol{x}, \boldsymbol{y}) \in \Sigma \times \gamma}\left|\boldsymbol{H}(\boldsymbol{x}, \boldsymbol{y})-\boldsymbol{H}_{p}(\boldsymbol{x}, \boldsymbol{y})\right| \leq M^{\prime \prime} \zeta^{\left(2 p^{*}+2\right)} \leq M^{\prime \prime \prime} \zeta^{(p+1)} .
$$

\footnotetext{
${ }^{12} \mathrm{Be}$ aware that this estimate depends only on the regularity of $\boldsymbol{H}(\cdot, \cdot)$. It is well established today that the approximation of any analytical (or piecewise analytical) function by polynomials (or piecewise polynomials) yields an exponential convergence rate.
} 
Now defining $\left(\mathcal{B}_{\Gamma}\right)_{p}$ like in (4.6) after replacing the kernel $\boldsymbol{H}(\cdot, \cdot)$ by $\boldsymbol{H}_{p}(\cdot, \cdot)$, we obtain a linear operator on $L^{2}(\Sigma)$ with a rank $\leq p$. Cauchy-Schwarz' inequality provides

$$
\left\|\left(\mathcal{B}_{\Sigma}-\left(\mathcal{B}_{\Sigma}\right)_{p}\right) \lambda\right\|_{\mathcal{L}\left(L^{2}(\Sigma)\right)} \leq\left\|\left[\chi_{\lambda}\right]\right\|_{L^{2}(\gamma)}\left\|\boldsymbol{H}-\boldsymbol{H}_{p}\right\|_{L^{2}(\gamma \times \Sigma)}, \quad \forall \lambda \in L^{2}(\Sigma) .
$$

The stability of the problem on $\chi_{\lambda}$ together with the estimate (5.6) conclude to

$$
\left\|\mathcal{B}_{\Sigma}-\left(\mathcal{B}_{\Sigma}\right)_{p}\right\|_{\mathcal{L}\left(L^{2}(\Sigma)\right)} \leq b \zeta^{p}
$$

The proof is complete owing to (5.1).

Proposition 5.2. Let $d=3$ and $K(\cdot)=1$. Assume that the fictitious boundary $\Sigma$ is Lipschitz-regular and that it can be separated form $\gamma$ by an ellipsoid. It holds that

$$
\mu_{p} \leq b \zeta^{\sqrt{p}}, \quad \forall p \in \mathbb{N} .
$$

$b$ is a positive constant and $\zeta \in] 0,1[$.

Proof. Let $\Pi$ be an ellipsoid that separates $\gamma$ from $\Sigma$. Only to fix the ideas we may work on a sphere modulo an easy changing of the variables. Given that $\Sigma, \Pi$ and $\Gamma$ are all compact sets we may construct a couple of spheres, $\Pi_{\Sigma}$ the smallest one surrounding $\Sigma$ and $\Pi_{\Gamma}$ the largest one encircled by $\Gamma$. The sphere $\Pi$ is then immersed in the annular domain defined by $\Pi_{\Sigma}$ and $\Pi_{\Gamma}$. We denote by $(\varrho, R)$ the radii of both spheres $\left(\Pi_{\Sigma}, \Pi_{\Gamma}\right)$. Now, the proof follows basically the previous one. We only mention the modifications due to the third dimension.

Let $\left(Y_{\ell}^{m}\right)_{\ell \geq 0,-\ell \leq m \leq \ell}$ be the spherical harmonics. Any given $\boldsymbol{x} \in \Sigma$ may be referred to by $r \hat{\boldsymbol{x}}$ with $r=|\boldsymbol{x}|$. The harmonicity of $\boldsymbol{H}(\cdot, \boldsymbol{y})$ outside the sphere $\Pi$ yields the expansion

$$
\boldsymbol{H}(\boldsymbol{x}, \boldsymbol{y})=\sum_{\ell=0}^{\infty} \sum_{m=-\ell}^{\ell}\left(\boldsymbol{a}_{\ell}^{m}(\boldsymbol{y}) r^{\ell}+\boldsymbol{b}_{\ell}^{m}(\boldsymbol{y}) r^{-\ell-1}\right) Y_{\ell}^{m}(\hat{\boldsymbol{x}}) .
$$

The coefficients $\left(\boldsymbol{a}_{\ell}^{m}(\boldsymbol{y}), \boldsymbol{b}_{\ell}^{m}(\boldsymbol{y})\right)_{\ell, m}$ are computed through the following integrals and hence bounded as follows

$$
\max _{\boldsymbol{y} \in \gamma}\left|\boldsymbol{a}_{\ell}^{m}(\boldsymbol{y})\right| \leq M^{\prime} R^{-\ell}, \quad \max _{\boldsymbol{y} \in \gamma}\left|\boldsymbol{b}_{\ell}^{m}(\boldsymbol{y})\right| \leq M^{\prime} \varrho^{\ell+1} .
$$

Let $p$ be a given integer and $p^{*}$ be the integer part of $(\sqrt{p}-1)$. We define the truncated kernel by

$$
\boldsymbol{H}_{p}(\boldsymbol{x}, \boldsymbol{y})=\sum_{\ell=0}^{p^{*}} \sum_{m=-\ell}^{\ell}\left(\boldsymbol{a}_{\ell}^{m}(\boldsymbol{y}) r^{\ell}+\boldsymbol{b}_{\ell}^{m}(\boldsymbol{y}) r^{-\ell-1}\right) Y_{\ell}^{m}(\hat{\boldsymbol{x}}) .
$$

The estimate of the error on the kernel $\boldsymbol{H}(\cdot, \cdot)$ is provided by

$$
\max _{\boldsymbol{y} \in \gamma}\left|\boldsymbol{H}(\boldsymbol{x}, \boldsymbol{y})-\boldsymbol{H}_{p}(\boldsymbol{x}, \boldsymbol{y})\right| \leq M^{\prime} \sum_{\ell=p^{*}+1}^{\infty}(2 \ell+1)^{2}\left(\frac{r}{R}\right)^{\ell}+M^{\prime} \sum_{\ell=p^{*}+1}^{\infty}(2 \ell+1)^{2}\left(\frac{r}{\varrho}\right)^{-\ell-1} .
$$

We used here the bound $\left(2 \max \left|Y_{\ell}^{m}(\cdot)\right|\right) \leq(2 \ell+1)$ (see [10]). Defining the parameter $\zeta \in[0,1[$ as in (5.5), the bound can be expressed as:

$$
\max _{(\boldsymbol{x}, \boldsymbol{y}) \in \Sigma \times \gamma}\left|\boldsymbol{H}(\boldsymbol{x}, \boldsymbol{y})-\boldsymbol{H}_{p}(\boldsymbol{x}, \boldsymbol{y})\right| \leq M^{\prime} \sum_{\ell=p^{*}+1}^{\infty}(2 \ell+1)^{2} \zeta^{\ell+1} \leq M^{\prime \prime} \zeta^{(\sqrt{p})} .
$$

Remarking that the rank of $\left(\mathcal{B}_{\Sigma}\right)_{p}$ defined by the kernel $\boldsymbol{H}_{p}(\cdot, \cdot)$ is $\leq p$ and using (5.1) completes the proof. 
Remark 5.1. The proof may be proceeded following similar lines with some necessary adding for an arbitrary $K(\cdot)$ provided that $\Sigma$ is chosen regular and the Green function $G(\boldsymbol{x}, \cdot)$ is smooth at the vicinity of $\Sigma$. The approximation of that Kernel on the complete orthogonal system in $L^{2}(\Sigma)$ of the eigenvectors of the LaplaceBeltrami operator yields exponential accuracy (see [10,30]).

Both propositions allow to state the final convergence result of the GMRES method in two and three dimensions.

Theorem 5.3. Let $K(\cdot)=1$. Assume that the fictitious boundary $\Sigma$ is Lipschitz-regular. Then, GMRES converges and we have that

$$
\left(\left\|r_{m}\right\|_{L^{2}(\Sigma)}\right)^{1 / m} \leq b \zeta^{m} \quad \forall m \in \mathbb{N} .
$$

The convergence rate in three dimensions is as follows

$$
\left(\left\|r_{m}\right\|_{L^{2}(\Sigma)}\right)^{1 / m} \leq b \zeta^{\sqrt{m}} \quad \forall m \in \mathbb{N} .
$$

In both estimates, $\zeta \in] 0,1[$ and decreases when the distance between $\Sigma$ and $\gamma$ grows.

Proof. Combining the abstract result of Lemma 4.1 and Proposition 5.1 yields that

$$
\left\|r_{m}\right\|_{L^{2}(\Sigma)} \leq \prod_{p=0}^{m-1} b \zeta^{p}=b^{m} \zeta^{(m-1) m / 2}
$$

hence the proof in two dimensions (after the necessity to change the value of $\zeta$ ). The three dimensions is handled in the same way owing to Proposition 5.2 and the final bound is due to $\sum_{p=0}^{m-1} \sqrt{p}=\mathcal{O}(m \sqrt{m})$.

Remark 5.2. When a finite element approximation is intended, the discrete operator $\left(\mathcal{B}_{\Sigma}\right)_{h}$ fulfills similar properties as $\mathcal{B}_{\Sigma}$ when the meshes in $\omega_{\gamma}$ and in $\Omega_{\gamma}$ are nested. In particular, its spectral radius remains lower than one and then the global operator $\left(\mathcal{H}_{\Sigma}\right)_{h}=\left(\mathcal{I}_{h}+\left(\mathcal{B}_{\Sigma}\right)_{h}\right)$ is but a small perturbation of the identity and is therefore well conditioned. Moreover, given that $\left(\mathcal{B}_{\Sigma}\right)_{h}$ is a good approximation of $\left(\mathcal{B}_{\Sigma}\right)$ for small $h$, we shall obtain similar convergence rates of GMRES in the discrete level. Numerical evidences will confirm this claim.

\section{Perforated domains}

In many real life situations we may be concerned with partial differential equations set in domains with a simple shape that contain a set of Lipschitzian holes which may dramatically affect the efficiency of the computations by standard discretizations methods. Several models are listed in [28] (see also [22]) for which the domain is perforated, such as thermal conductivity for composite materials or time-discretized fluid-particle flow problems.

We aim the extension of the TOS method to the Poisson problem set on a perforated domain. The holes are assumed to be distant from each other and to simplify the presentation, we consider that $\Omega_{\gamma}$ contains a single hole represented by $\Omega_{\gamma}^{\prime}$. The generalization to multiple holes is straightforward. Denote by $\Omega=\Omega_{\gamma} \cup \Omega_{\gamma}^{\prime}$, called the safe domain (see Fig. 2) and $\gamma$ is the common boundary between $\Omega_{\gamma}$ and $\Omega_{\gamma}^{\prime}$. We need also the notation $H_{*}^{1}(\Omega)=H^{1}\left(\Omega_{\gamma}^{\prime}\right) \times H_{\Gamma}^{1}\left(\Omega_{\gamma}\right)$, the broken Sobolev space with order one.

Let $f$ be given in $L^{2}\left(\Omega_{\gamma}\right)$, the Laplace problem is written as: find $\varphi \in \mathcal{D}^{\prime}\left(\Omega_{\gamma}\right)$ such that

$$
\begin{aligned}
-\operatorname{div}(K \nabla \varphi) & =f & & \text { in } \Omega_{\gamma} \\
\varphi & =0 & & \text { on } \Gamma \\
\left(K \partial_{\boldsymbol{n}}\right) \varphi & =0 & & \text { in } \gamma .
\end{aligned}
$$



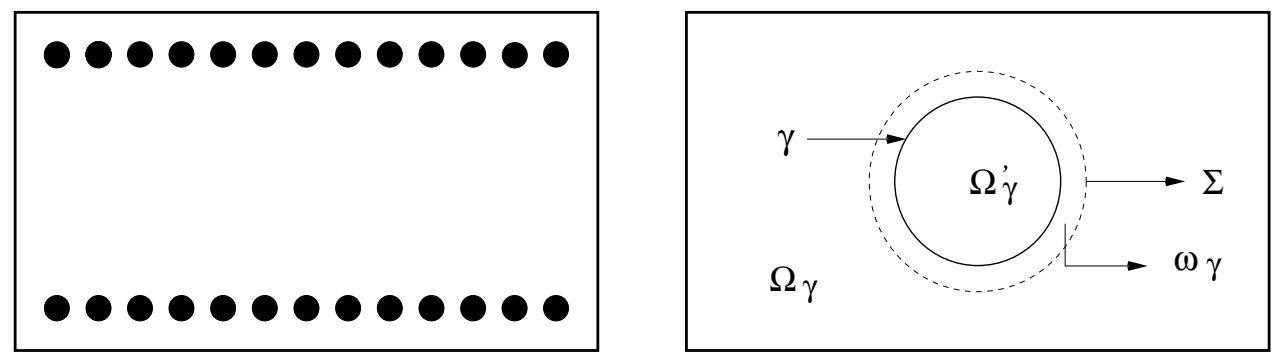

FigurE 2. The perforated domain.

We introduce a boundary $\Sigma$ that has no common points with $\gamma$. Denote by $\omega_{\gamma}$ the "crust-like" domain delimited by $\Sigma$ and $\gamma$. Problem (1.1)-(1.3) may be expressed through two subproblems. The first, with transmission conditions, is set on the safe domain $\Omega$ : find $\eta \in H_{*}^{1}(\Omega)$ such that

$$
\begin{aligned}
-\operatorname{div}(K \nabla \eta) & =\tilde{f} & & \text { in } \Omega_{\gamma} \cup \Omega_{\gamma}^{\prime}, \\
{[\eta] } & =\chi & & \text { on } \gamma, \\
{\left[\left(K \partial_{n}\right) \eta\right] } & =0 & & \text { on } \gamma, \\
\eta & =0 & & \text { on } \Gamma .
\end{aligned}
$$

$\tilde{f}$ is the extension of $f$ by zero in $\Omega_{\gamma}^{\prime}$. The second subproblem is defined in $\omega_{\gamma}$ and consists in: find $\chi \in H^{1}\left(\omega_{\gamma}\right)$ such that

$$
\begin{aligned}
-\operatorname{div}(K \nabla \chi) & =f & & \text { in } \omega_{\gamma} \\
\chi & =\eta & & \text { on } \Sigma \\
\left(K \partial_{n}\right) \chi & =0 & & \text { on } \gamma .
\end{aligned}
$$

Notice that the difference with the cracked domain resides in the transmission condition (6.5), where $\chi$ is used instead of $[\chi]$. The equivalence can be checked as follows. Extend $\chi$ by zero in $\Omega_{\gamma}^{\prime}$ to obtain $\tilde{\chi}$. Then, look at $(\eta-\tilde{\chi})$. It is harmonic in $\Omega_{\gamma}^{\prime} \cup \omega_{\gamma}$, its trace and its normal derivative are continuous across $\gamma$ and it vanishes at $\Sigma$. We deduce that $\tilde{\chi}=\eta$ in $\Omega_{\gamma}^{\prime} \cup \omega_{\gamma}$ from which we infer that $\eta=\varphi$ in $\Omega_{\gamma}$ and $\eta=0$ in $\Omega_{\gamma}^{\prime}$. Moreover we have that $\chi=\varphi$ in $\omega_{\gamma}$.

Total Overlapping Schwarz' method can be addressed now. Assume $\left(\chi^{m}, \eta^{m}\right)$ is known, $\eta^{m+1} \in H_{*}^{1}(\Omega)$ is solution of

$$
\begin{aligned}
-\operatorname{div}\left(K \nabla \eta^{m+1}\right) & =\tilde{f} & & \text { in } \Omega_{\gamma} \cup \Omega_{\gamma}^{\prime}, \\
{\left[\eta^{m+1}\right] } & =\chi^{m} & & \text { on } \gamma, \\
{\left[\left(K \partial_{\boldsymbol{n}}\right) \eta^{m+1}\right] } & =0 & & \text { on } \gamma, \\
\eta^{m+1} & =0 & & \text { on } \Gamma,
\end{aligned}
$$

and $\chi^{m+1} \in H^{1}\left(\omega_{\gamma}\right)$ satisfies

$$
\begin{aligned}
-\operatorname{div}\left(K \nabla \chi^{m+1}\right) & =f & & \text { in } \omega_{\gamma} \\
\chi^{m+1} & =\eta^{m+1} & & \text { on } \Sigma \\
\left(K \partial_{n}\right) \chi^{m+1} & =0 & & \text { on } \gamma .
\end{aligned}
$$


That the recurrence is well defined is readily checked and we get two sequences $\left(\chi^{m}\right)_{m} \subset H^{1}\left(\omega_{\gamma}\right)$ and $\left(\eta^{m}\right)_{m} \subset$ $H_{*}^{1}(\Omega)$. The convergence study can be developed following the same lines as for the cracked domains and yields a linear convergence similar to Proposition 2.1.

Remark 6.1. The derivation of the condensed equation on $\Sigma$ can be made as in Section 3. Using a Krylov method enhances the convergence speed versus the Schwarz algorithm and the estimates of Theorem 5.3 are valid as well for perforated domains.

\section{Some implementation hints}

We are left here with the finite element approximation of the coupled problem we are involved in, in view of their implementation. After the discretization of equations (1.4)-(1.7) and (1.8)-(1.10), we show how to generate the associated algebraic system. We operate by then the decoupling process by means of the TOS method. Let us make it clear right away, we will not address the implementation of the problem (1.4)-(1.7) when $\eta_{\mid \Sigma}$ is given nor that of (1.8)-(1.10) if $[\chi]_{\mid \gamma}$ is available. These are all well known tasks (see, e.g., [26]). We do rather dedicate our attention to the coupling/uncoupling features and give some tips for their programming.

Let us construct a regular triangulation $\mathcal{T}_{h}$ of $\Omega_{\gamma}$ so that the crack is the union of segments or faces of some $K \in \mathcal{T}_{h}$. The size of the mesh is $h$. Here, we shall assume that the triangulation is compatible with the $\omega_{\gamma}$ that is $\Sigma$ follows some lines of the mesh so that the restriction of $\mathcal{T}_{h}$ to $\omega_{\gamma}$ results in a full mesh ${ }^{13}$. Denote by $V_{h}$, the finite element space

$$
V_{h}\left(\Omega_{\gamma}\right)=\left\{\psi_{h} \in \mathcal{C}\left(\Omega_{\gamma}\right) ; \quad\left(\psi_{h}\right)_{\mid K} \in \mathcal{P}_{p}(K), \quad \forall K \in \mathcal{T}_{h}\right\} \subset H^{1}\left(\Omega_{\gamma}\right)
$$

$\mathcal{P}_{p}$ is the set of polynomial with degree $p \geq 1$. Notice like those of $H^{1}\left(\Omega_{\gamma}\right)$, the functions of $V_{h}\left(\Omega_{\gamma}\right)$ are discontinuous across $\gamma$. We set $V_{h}\left(\omega_{\gamma}\right)=\left(V_{h}\left(\Omega_{\gamma}\right)\right)_{\mid \omega_{\gamma}}$, the space of the restriction of all the functions in $V_{h}\left(\Omega_{\gamma}\right)$ and define the subspace $V_{h, \Sigma}\left(\omega_{\gamma}\right)$ by incorporating the Dirichlet condition on $\Sigma$,

$$
V_{h, \Sigma}\left(\omega_{\gamma}\right)=\left\{\psi_{h} \in V_{h}\left(\omega_{\gamma}\right) ; \quad\left(\psi_{h}\right)_{\mid \Sigma}=0\right\} .
$$

We intend to compute the discrete solution $\left(\chi_{h}, \eta_{h}\right)$ of $(1.8)-(1.10)$ and $(1.4)-(1.7)$. We proceed like in Remark 1.7 and choose to eliminate $\eta_{h}$ and keep $\chi_{h}$ as the only unknown. As currently used, the boundary condition $(1.9)$ on $\left(\chi_{h}\right)_{\mid \Sigma}$ has to be lifted. It is accomplished as follows

$$
\chi_{h}=\underline{\chi}_{h}+\mathcal{R}_{h}^{\Sigma}\left(\eta_{h}\right) \in V_{h}\left(\omega_{\gamma}\right)
$$

with $\underline{\chi}_{h} \in V_{h, \Sigma}\left(\omega_{\gamma}\right)$. The operator $\mathcal{R}_{h}^{\Sigma}$ is the canonical discrete lifting in $V_{h}\left(\omega_{\gamma}\right)$ of finite elements traces on $\Sigma^{14}$. The discrete variational problem reads hence as: find $\underline{\chi}_{h} \in V_{h, \Sigma}\left(\omega_{\gamma}\right)$ such that

$$
\int_{\omega_{\gamma}} \nabla \underline{\chi}_{h} \nabla \psi_{h} \mathrm{~d} \boldsymbol{x}+\int_{\omega_{\gamma}} \nabla \mathcal{R}_{h}^{\Sigma}\left(\eta_{h}\right) \nabla \psi_{h} \mathrm{~d} \boldsymbol{x}=\int_{\omega_{\gamma}} f \psi_{h} \mathrm{~d} \boldsymbol{x}, \quad \forall \psi_{h} \in V_{h, \Sigma}\left(\omega_{\gamma}\right)
$$

Denote by $\left(\boldsymbol{x}_{i}\right)_{1 \leq i \leq i^{*}}$ the nodes of $\overline{\left(\omega_{\gamma}\right)^{15}}$. Indicating that $\boldsymbol{x}_{i}$ is located on $\Sigma(\gamma, \ldots)$ is conventionally made by $i \in \Sigma(i \in \gamma, \ldots)$. The shape functions of $V_{h}\left(\omega_{\gamma}\right)$ are $\left(\varphi_{i}\right)_{i \in \overline{\left(\omega_{\gamma}\right)}}$ (see [9]). The discrete solution $\underline{\chi}_{h}$ can be

\footnotetext{
13 For non-matching meshes, Hecht et al. observed in [21] that the convergence may be slowed because of the interpolation from one mesh to another. They suggest to add a harmonic patch step as in [19] that greatly increases the convergence rate. More numerical issues that may arise, for instance by the effect of quadrature integration, are successfully addressed in [21].

${ }^{14}$ The lifting that keeps unchanged the degrees of freedom on $\Sigma$ while the internal ones equal zero.

${ }^{15}$ The nodes on $\gamma$ give birth to two degrees of freedom.
} 
expanded as

$$
\underline{\chi}_{h}(\boldsymbol{x})=\sum_{i \in \overline{\left(\omega_{\gamma}\right)} \backslash \Sigma} \underline{\chi}_{h}(i) \varphi_{i}(\boldsymbol{x})=\sum_{i \in \overline{\left(\omega_{\gamma}\right)} \backslash \Sigma} \chi_{h}(i) \varphi_{i}(\boldsymbol{x}) .
$$

The degrees of freedom are given by $\chi_{h}(i)=\chi_{h}\left(\boldsymbol{x}_{i}\right)$. Let us call $\underline{\chi}_{h}$, the vector $\left(\underline{\chi}_{h}(i)\right)_{i \in\left(\overline{\left.\omega_{\gamma}\right)} \backslash \Sigma\right.}$. Similarly let $\underline{\boldsymbol{\eta}}_{h}^{\Sigma}$ be the vector $\left(\eta_{h}(i)\right)_{i \in \Sigma}$. The algebraic system related to the discrete problem (7.1) is hence given by

$$
\boldsymbol{A}_{h}+\boldsymbol{C} \boldsymbol{\eta}_{h}^{\Sigma}=\underline{\boldsymbol{F}}_{h}
$$

The vector $\underline{\boldsymbol{F}}_{h}$ describes the action of $f$ in (7.1). $\boldsymbol{A}=\left(a_{i, j}\right)_{i, j \in \overline{\left(\omega_{\gamma}\right)} \backslash \Sigma}$ is the stiffness matrix of the Laplacian with a Dirichlet condition on $\Sigma$. The rectangular matrix $C=\left(c_{i, \ell}\right)_{i \in \overline{\left(\omega_{\gamma}\right)} \backslash \Sigma, \ell \in \Sigma}$ is reduced to a small block. Indeed, as soon as the node $\boldsymbol{x}_{i}$ is not connected to another node located on $\Sigma$ the entry $c_{i, \ell}=0$. We handily write $i \in \bar{\Sigma}$ when $\boldsymbol{x}_{i}$ is connected to a node in $\Sigma$. A final step has to be accomplished to obtain the final equation. Observe that $\eta_{h}$, the discrete solution the transmission problem (1.4)-(1.7), depends on $\left[\chi_{h}\right]_{\mid \gamma}=\left[\underline{\chi}_{h}\right]_{\mid \gamma}$ and $f$. There holds that $\left(\eta_{h}\right)_{\mid \Sigma}=T\left(\underline{\chi}_{h}\right)+\left(\breve{\eta}_{h}\right)_{\mid \Sigma}$, where $\breve{\eta}_{h}$ is the finite element approximation of $\breve{\eta}$, the solution of (1.19)-(1.20). That equality may be translated into a matrix form,

$$
\boldsymbol{\eta}_{h}^{\Sigma}=\boldsymbol{T}\left(\underline{\chi}_{h}\right)+\breve{\eta}_{h}^{\Sigma}
$$

with $\boldsymbol{T}=\left(t_{k j}\right)_{k \in \gamma, j \in \overline{\left(\omega_{\gamma}\right)} \backslash \Sigma}$. Actually $\boldsymbol{T}$ turns to be substantially sparse given that $t_{k j}=0$ for all $j \notin \gamma$. Plugging it into the discrete equation yields the global system

$$
(\boldsymbol{A}+\boldsymbol{C T}) \underline{\underline{\chi}}_{h}=\underline{\boldsymbol{F}}_{h}-\boldsymbol{C} \breve{\boldsymbol{\eta}}_{h}^{\Sigma}
$$

Equation (7.2) is nothing else than the discretization of (1.21). $(\boldsymbol{A}, \boldsymbol{C}, \boldsymbol{T})$ are the approximation of the operators $(\mathcal{A}, \mathcal{C}, \mathcal{T})$. It is the one we implemented in MELINA, where $\boldsymbol{A}, \boldsymbol{C}$ so as $\underline{\boldsymbol{F}}_{h}$ are constructed during the preprocessing step. Let us underline that the discrete Laplacian $\boldsymbol{A}$ is sparse, symmetric and positive definite. The $\boldsymbol{C T}$ is reduced to a local block that governs the coupling between $\gamma$ to $\Sigma$. It is supported in the set of indices $(i, j) \in \bar{\Sigma} \times \gamma$ but has no particular symmetry. The structure of the global matrix is consequently altered. The solvers programmed in MELINA to invert the system (7.2) are (LU) method, some of its variants, and the Bi-Conjugate Gradient algorithm. The version of GMRES of [13] was combined to MELINA to realize the numerical experiences presented in the next section.

That the description of the coupled approach is achieved we now switch to the TOS procedure. The methodology exposed above can be repeated once again and results in the following equation

$$
\boldsymbol{A} \underline{\boldsymbol{\chi}}_{h}^{m+1}+(\boldsymbol{C} \boldsymbol{T}) \underline{\boldsymbol{\chi}}_{h}^{m}=\underline{\boldsymbol{F}}_{h}-\boldsymbol{C} \breve{\eta}_{h}^{\Sigma}
$$

or again

$$
\underline{\boldsymbol{\chi}}_{h}^{m+1}+(\boldsymbol{A})^{-1}(\boldsymbol{C} \boldsymbol{T}) \underline{\boldsymbol{\chi}}_{h}^{m}=(\boldsymbol{A})^{-1}\left(\underline{\boldsymbol{F}}_{h}-\boldsymbol{C} \breve{\boldsymbol{\eta}}_{h}^{\Sigma}\right) .
$$

We recognize here again the TOS-preconditioned form of the Richardson method. The preconditioner is $\boldsymbol{A}$. The advantage is to invert, a each iteration, a sparse symmetric system, the hope being that the convergence be attained in few iterations. The linear convergence of the TOS method proven in Proposition 2.1 tells in particular that the spectral radius of the iteration matrix $(\boldsymbol{A})^{-1}(\boldsymbol{C T})$ is lower than unity and is expected to decrease with the inverse of the distance between $\Sigma$ and the crack $\gamma$.

Remark 7.1. The vector $\underline{\chi}_{h}^{m}$ provides the $\chi_{h}^{m}\left(\boldsymbol{x}_{i}\right)$ for $i \in \overline{\left(\omega_{\gamma}\right)} \backslash \Sigma$. To complete the construction of $\chi_{h}^{m}$ we need to compute $\chi_{h}^{m}\left(\boldsymbol{x}_{i}\right)$ for $i \in \Sigma$. This is obtained as follows

$$
\chi_{h}^{m}\left(\boldsymbol{x}_{i}\right)=\eta_{h}^{m-1}\left(\boldsymbol{x}_{i}\right)=\left((\boldsymbol{C} \boldsymbol{T}) \underline{\boldsymbol{\chi}}_{h}^{m-1}\right)(i) .
$$


Remark 7.2. A guidance for the discretization of (3.3) follows the same lines with some necessary adaptations. In addition, one can check also that $\lambda_{h}^{m}$ coincides exactly with $\left(\chi_{h}^{m}\right)_{\mid \Sigma}$.

\section{ILLUSTRATIONS AND NUMERICAL RESUlTS}

We consider a simple circular geometry for which analytical computations are accessible. The aim is to look at the spectrum of the operator $\mathcal{B}_{\Sigma}$ which is the crux of the convergence study of the modified Schwarz algorithm so as of the Residual Krylov Subspaces methods. We check the exponential decaying of the singular values of it. Then, we provide some numerical experiments illustrating the convergence of TOS and GMRES methods.

\subsection{Analytical calculus}

Assume that the domain $\Omega_{\gamma}$ is annular in $\mathbb{R}^{2}$, centered at the origin with double radius $(1, R)$. The boundary $\Gamma$ is then the circle with radius $R$ and $\gamma$ the concentric circle with radius 1 . The fictitious circular boundary $\Sigma$ is concentric to both $\Gamma$ and $\gamma$ with radius $\varrho(1<\varrho<R)$. The geometric symmetry suggests that the eigen-modes of $\mathcal{B}_{\Sigma}$ are provided by the Fourier modes $(\cos (k \theta))_{k \geq 1}$. The calculation of the eigenvalue related to $\lambda=\cos (k \theta)$ requires the determination of $\chi_{\lambda}$ solution of (6.8)-(6.10) where $f=0$ in (6.8) and $\chi_{\lambda}=\lambda$ instead of (6.9). Then we compute $\eta_{\lambda}$ satisfying the transmission problem (6.4)-(6.7) where $\tilde{f}=0$ and $\left[\eta_{\lambda}\right]=\chi_{\lambda}$ instead of (6.6). The overall calculus are realized for both problems by means of the method of the variables separation. We obtain that

and

$$
\chi_{\lambda}(\boldsymbol{x})=\frac{r^{k}+r^{-k}}{\varrho^{k}+\varrho^{-k}} \cos (k \theta),
$$

$$
\eta_{\lambda}(\boldsymbol{x})= \begin{cases}-\frac{\left(R^{-2 k}+1\right) r^{k}}{\varrho^{k}+\varrho^{-k}} \cos (k \theta) & 0 \leq r \leq 1, \\ -\frac{R^{-2 k} r^{k}-r^{-k}}{\varrho^{k}+\varrho^{-k}} \cos (k \theta) & 1 \leq r .\end{cases}
$$

The eigenvalues of $\mathcal{B}_{\Sigma}$ are then given by

$$
\mu_{k}=\frac{\varrho^{-2 k}-R^{-2 k}}{1+\varrho^{-2 k}}
$$

The eigenvalues decay exponentially fast towards zero and the decreasing rate depends on the thickness $(\varrho)$ of the crust-like domain $\omega_{\gamma}$. For the exterior Poisson problem $(R=\infty)$ we have

$$
\mu_{k}=\frac{\varrho^{-2 k}}{1+\varrho^{-2 k}} .
$$

Similar computations can be achieved in $\mathbb{R}^{3}$, the harmonics spherics $\left(Y_{\ell}^{m}(\theta, \phi)\right)_{m \geq 0,-m \leq \ell \leq m}$ are used instead of the Fourier modes. The eigenvalues of $\mathcal{B}_{\Sigma}$ are then provided by

$$
\mu_{\ell}^{m}=\frac{\varrho^{-(2 \ell+1)}-R^{-(2 \ell+1)}}{\left(1+\ell^{-1}\right)+\varrho^{-(2 \ell+1)}} .
$$

Sorting them in an increasing order with a single index $p=(m+1)+\ell(\ell+1)$ we obtain that $\mu_{p}=\mathcal{O}\left(\varrho^{(-2 \sqrt{p})}\right)$. According to Proposition 5.2, these are expected trends.

Remark 8.1. The iterating operator $\mathcal{B}_{\Sigma}$ for the version of the Schwarz algorithm analyzed in [24] has the following eigenvalues

$$
\mu_{k}=\frac{2\left(\varrho^{-2 k}-R^{-2 k}\right)}{\left(1-R^{-2 k}\right)\left(1+\varrho^{-2 k}\right)} .
$$


When the external radius $R$ grows to infinity which corresponds to exterior problems, we obtain

$$
\mu_{k}=\frac{2 \varrho^{-2 k}}{1+\varrho^{-2 k}} .
$$

The correction added to the Schwarz method produces an algorithm almost twice faster in addition to the implication it has on the computation of the iterates $\left(\eta^{m}\right)_{m}$, that turns out to be cheaper.

\subsection{Numerical discussion}

We assess the TOS method for the Poisson problem in cracked and perforated unbounded domains through three numerical experiences. The finite element approximation of $\left(\chi^{m}\right)_{m}$ and the computation of $\left(\eta^{m}\right)_{m}$ are obtained by MELINA (see [27]). It was necessary to implement new procedures dedicated to TOS method. The meshes in $\omega_{\gamma}$ and $\Omega_{\gamma}$ are generated by means of the EMC2 program (see [20]).

We first address the computation of the 'electrical' potential created by a quadrupole constructed by superimposing two dipoles with parallel moments. The four conductors are circular with radius 0.3 and they are centered at points $( \pm 1, \pm 1)$. Their union gives the internal $\Omega_{\gamma}^{\prime}$ and $\gamma=\partial \Omega_{\gamma}^{\prime}$. The safe domain is the whole plane $\mathbb{R}^{2}$ and the perforated domain $\Omega_{\gamma}=\mathbb{R}^{2} \backslash \Omega_{\gamma}^{\prime}$. The electrical charges in the conductors are concentrated along the surface with a piecewise constant density $\rho$. The north and east poles are positively charged so that $\rho=1$ while the south and west poles are negatively charged to $\rho=-1$. We have therefore to solve the exterior Poisson problem on a perforated domain $\Omega_{\gamma}$,

$$
\begin{array}{rll}
(-\Delta) \varphi=0 & & \text { in } \Omega_{\gamma} \\
\varphi=0 & & \text { at } \infty \\
\partial_{\boldsymbol{n}} \varphi=\rho & & \text { in } \gamma .
\end{array}
$$

This is similar to the problem (6.1)-(6.3) where $K(\cdot)=1$ and the boundary $\Gamma$ is located at infinity. Notice that when writing down the TOS method following (6.11)-(6.14) and (6.15)-(6.17), the advantages are even substantially increased since the problem (6.11)-(6.14) is explicitly solved by means of the appropriate integral representation to obtain $\left(\eta^{m+1}\right)$, as indicated in Remark 1.5. Procedures for the computation of the integral (1.18) are available in MELINA with a highly accurate quadrature formulas (see [27]).

We realize two experiences. One is related to a connected $\omega_{\gamma} . \Sigma$ is the union of the edges of the square centered at the origin with edges of length 4 unities and $\omega_{\gamma}$ is that square minus the quadrupole. The second calculation corresponds to a 4-connected interface $\Sigma$. Each connected component is a circle (concentric to a conductor) of radius 0.5 and each connected component of $\omega_{\gamma}$ is therefore the union of the four annular domains with double radius $(0.3,0.5)$. The symmetries with respect to both $x_{1^{-}}$and $x_{2}$-axis are taken into account during the simulation so that the computations are run only on a the north-eastern quarter part of the domain. The equipotentials computed by the TOS method are depicted within the domain $\omega_{\gamma}$ in Figure 3. Both computations reproduce similar equipotentials at the vicinity of the electrical conductors. We measure the difference between both potentials to approximately $10^{-4}$ (in $\omega_{\gamma}$ ). More calculations are run when the $x_{2^{-}}$ symmetry of the quadrupole is broken by slightly moving the vertical conductors downward by a distance $=0.4$. The simulations are achieved on the whole domain $\omega_{\gamma}$ this time. Overall indicators and observations described for the symmetric quadrupole are also valid here (see Fig. 4). The curves of Figure 5, representing the residual versus the iterations, illustrate the linear convergence of the TOS method.

The second experience is involved in a cracked domain. We again compute the electrical potential by the same non-symmetric quadrupole where the conductors are flattened as in Figure 6. To exaggerate the jumps of the potential across the conductors we assume them charged with different densities on both sides. The charge density is $\rho=2$ on the east side of the northern conductor and $\rho=1$ on the west side. Along the eastern conductor $\rho$ is 2 along the north side and 1 along the south side. The densities of the other conductors are obtained by an odd symmetry with respect to the center of the quadrupole $(\rho=-2$ or $\rho=-1)$. Let us notice 

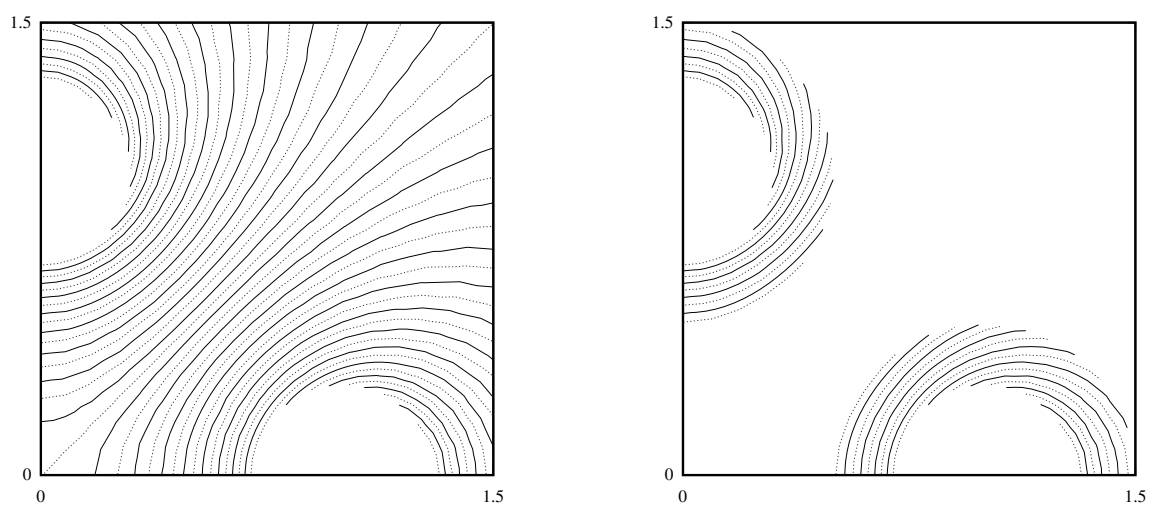

Figure 3. Equipotentials of the symmetric circular quadrupole (in $\left.\omega_{\gamma}\right)$.
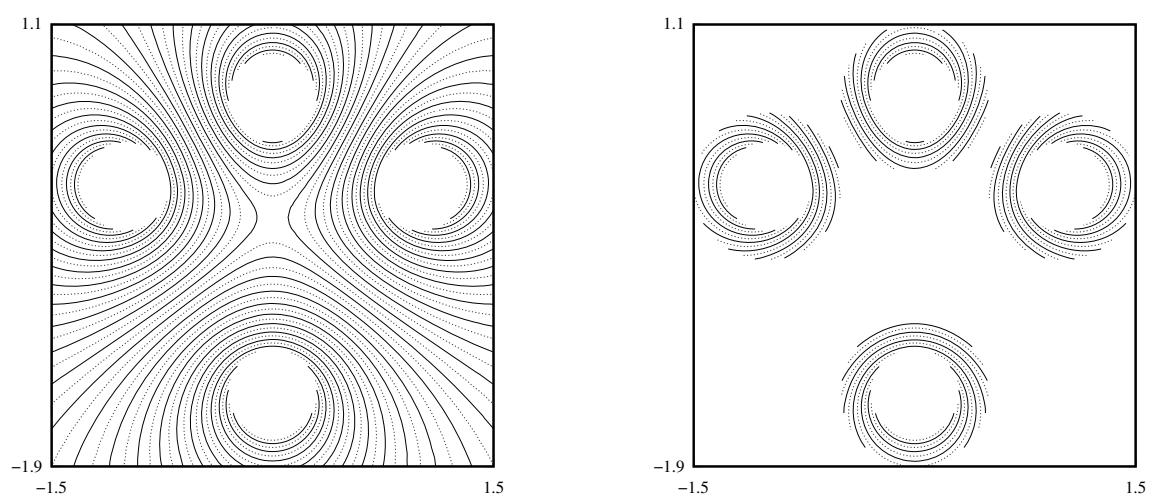

FiguRE 4. The non-symmetric circular quadrupole.
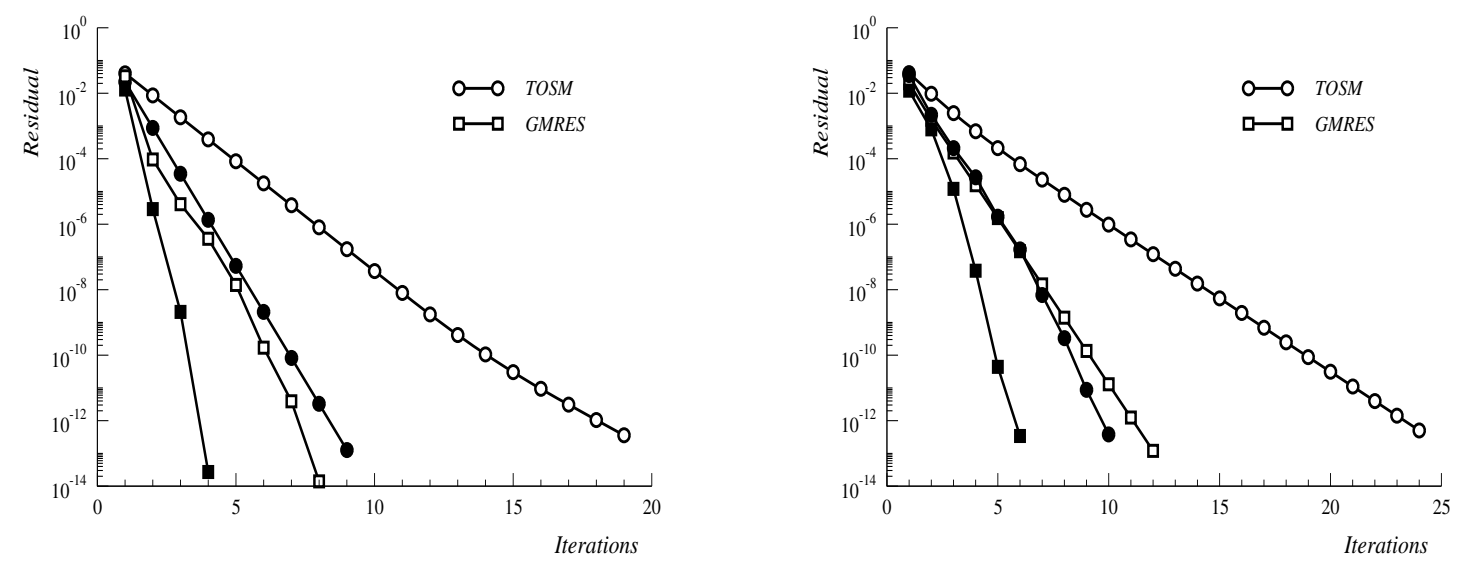

Figure 5. Convergence curves for the quadrupole. The symmetric quadrupole is represented in the left diagram and the non-symmetric one in the right diagram. Full symbols are used for connected $\omega_{\gamma}$ while empty symbols are for non-connected $\omega_{\gamma}$. Circular symbols correspond to the TOS method and squared symbols are for the GMRES. 

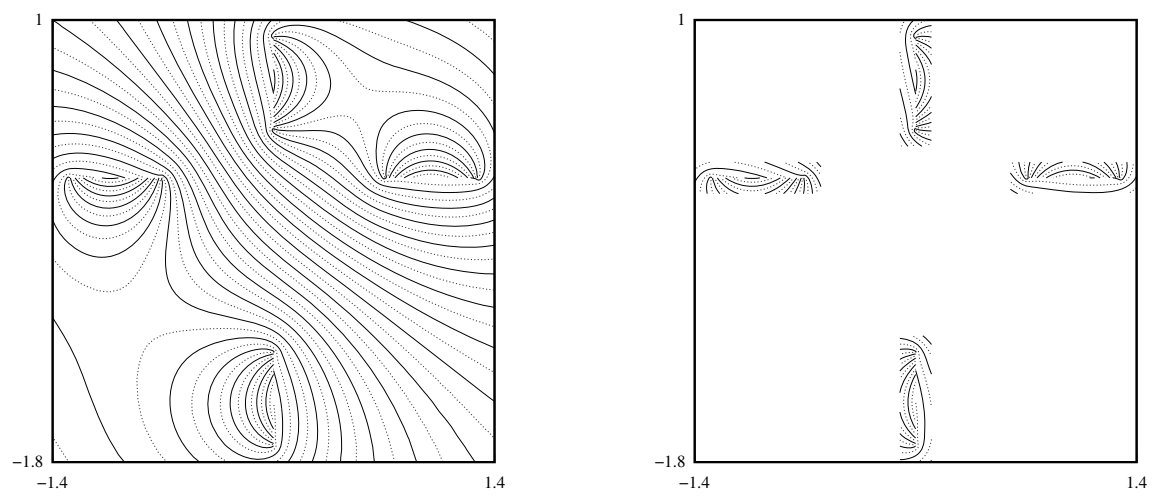

Figure 6. Equipotentials of the non-symmetric flat quadrupole. Charges concentration are higher along the sides where equipotentials are more dense.
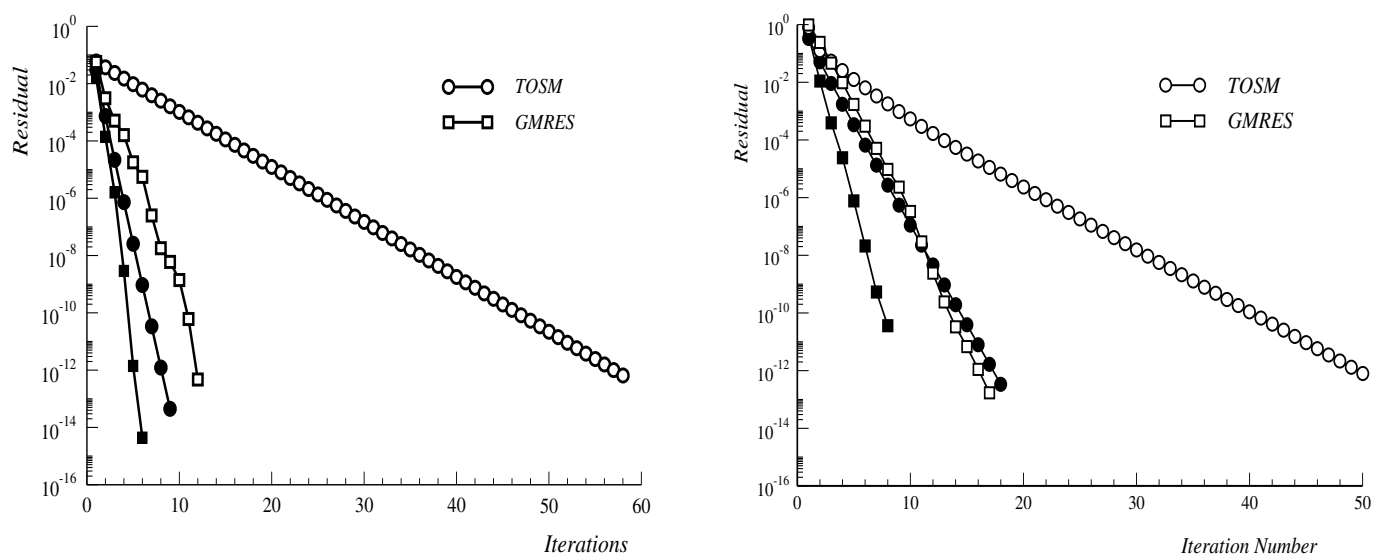

FIgURE 7. The TOS method and GMRES convergence curves: the flattened quadrupole to the left, the non-homogeneous quadrupole to the right.

that the non-connected computational domain is composed of elongated rectangles that fit the geometry of the conductors. The equipotentials represented in there show the jumps of the potential across the cracks. This has no effect on the behavior of the TOS method as illustrated by the convergence curve in Figure 7 . Indeed, the convergence rate is linear and decreases when the computational domain grows in size.

We also provide in Figure 7 the convergence curves for the preconditioned GMRES method for the nonsymmetric flat quadrupole. It is noticeable that unless the sub-domain $\omega_{\gamma}$ is very thin the TOS method by itself ensures a reasonable convergence rate and is an afforded tool to zoom the calculations at the vicinity of the cracks.

We perform the last experience in a medium with a discontinuous permettivity $K$ to figure out how the TOS method and GMRES behave. The non-symmetric quadrupole considered in the first test is immersed in a two layered medium. Each of the conductors is enclosed by a concentric annular medium with $K=0.25$ while the permettivity of the surrounding medium is $K=1$. Equipotentials are represented in Figure 8 for both connected and non connected $\omega_{\gamma}$. The convergence curves are depicted in the right diagram of Figure 7. We observe the expected convergence speed of the TOS method. As for the preconditioned GMRES method, the convergence is obtained in a few iterations so that one could hardly observe the asymptotic behavior of the algorithm as predicted in Theorem 5.3. 

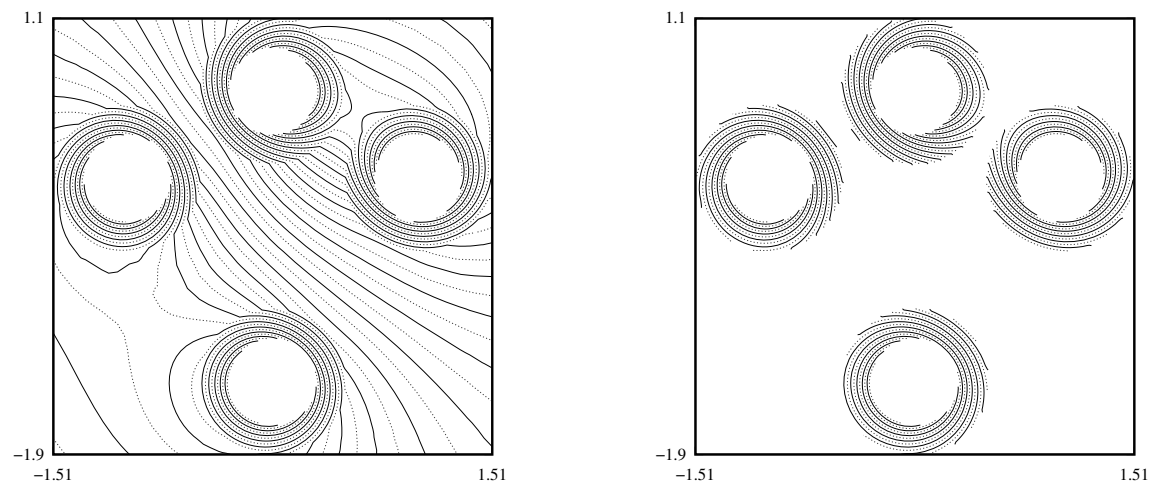

Figure 8. Equipotentials of the quadrupole immersed in a non-homogeneous medium.

\section{Conclusion}

We consider the particular adaptation of the Schwarz method with a Total Overlap, introduced in [3], by reformulating the transmission conditions that glue the subregions to each other. The advantages are multiform. The algorithm under interest is more competing than the original overlapping Schwarz method (even with a complete overlap) since it may be twice faster as illustrated by the example of Remark 8.1. Economically, the sub-problem, set in the whole domain, is most often well conditioned and may be solved rapidly, especially for simple shaped domains (see [28]). This algorithm may be used as a numerical zooming device in the regions of small scales, we refer to $[2,21]$ for some applications of a similar (Chimera Schwarz) approach in fluid mechanics. We are also involved in the TOS method used as a preconditioner for Minimum Residual Krylov Sub-spaces methods. The results established for the preconditioned GMRES show a super linear convergence which is assessed by some analytical and numerical calculations. Similar convergence estimates are expected to hold for variational type discretizations, though they arise many challenging interrogations with the subjacent matching grids issue. Tips and hints for numerical implementation may be found in [21] for the Zooming Chimera-Schwarz method. The ideas exposed in the present work may be applied as well to non-linear cracks where unilateral Signorini conditions are prescribed. Stating linear convergence results for the TOS method is aimed in a future work.

\section{Two TECHNICAL LEMMAS}

We prove that the operator $\mathcal{H}_{\Sigma}=\left(\mathcal{I}+\mathcal{B}_{\Sigma}\right)$ defined in $(3.2)$ is an isomorphism on $H^{1 / 2}(\Sigma)$. Since $\mathcal{B}_{\Sigma}$ is compact, using the Fredholm Alternative we deduce that the $\mathcal{H}_{\Sigma}$ has a closed range in $H^{1 / 2}(\Sigma)$. If we show that the kernel of the adjoint operator $\left(\mathcal{H}_{\Sigma}\right)^{*}$, defined in $H^{-1 / 2}(\Sigma)$, is reduced to the trivial $\{0\}$, we conclude that $\mathcal{H}_{\Sigma}$ is an isomorphism on $H^{1 / 2}(\Sigma)$. Let be given $\mu \in H^{-1 / 2}(\Sigma)$, we denote $\eta_{\mu} \in H^{1}(\Omega)$ the unique solution of

$$
\begin{aligned}
-\operatorname{div}\left(K \nabla \eta_{\mu}\right) & =0 & & \text { in } \Omega \backslash \Sigma, \\
{\left[\eta_{\mu}\right] } & =0 & & \text { on } \Sigma, \\
{\left[\left(K \partial_{n}\right) \eta_{\mu}\right] } & =\mu & & \text { on } \Sigma, \\
\eta_{\mu} & =0 & & \text { on } \Gamma,
\end{aligned}
$$

and define $\chi_{\mu} \in H^{1}\left(\omega_{\gamma}\right)$ such that

$$
\begin{aligned}
-\operatorname{div}\left(K \nabla \chi_{\mu}\right) & =0 & & \text { in } \omega_{\gamma} \\
\chi_{\mu} & =0 & & \text { on } \Sigma \\
\left(K \partial_{\boldsymbol{n}}\right) \chi_{\mu} & =\left(K \partial_{\boldsymbol{n}}\right) \eta_{\mu} & & \text { on } \gamma^{+} \cup \gamma^{-} .
\end{aligned}
$$


It can be checked by Green's formula that

$$
\left(\mathcal{B}_{\Sigma}\right)^{*} \mu=\left(\left(K \partial_{\boldsymbol{n}}\right) \chi_{\mu}\right)_{\mid \Sigma}
$$

We are in position to state the following.

Lemma 10.1. The linear operator $\mathcal{H}_{\Sigma}=\left(\mathcal{I}+\mathcal{B}_{\Sigma}\right)$ is an isomorphism on $H^{1 / 2}(\Sigma)$.

Proof. Let $\mu$ be in the kernel $\mathcal{N}\left(\left(\mathcal{H}_{\Sigma}\right)^{*}\right)$ then $\mu+\left(K \partial_{\boldsymbol{n}}\right) \chi_{\mu}=0$ on $\Sigma$. Extend $\chi_{\mu}$ trivially to the domain $\Omega_{\gamma}$ and still denote $\chi_{\mu}$ this extension. Then, consider $\zeta_{\mu}=\left(\eta_{\mu}-\chi_{\mu}\right)$; it is solution of

$$
\begin{array}{rll}
-\operatorname{div}\left(K \nabla \zeta_{\mu}\right)=0 & & \text { in } \Omega_{\gamma}, \\
\zeta_{\mu}=0 & & \text { on } \Gamma, \\
\left(K \partial_{n}\right) \zeta_{\mu}=0 & & \text { on } \gamma^{+} \cup \gamma^{-} .
\end{array}
$$

We have therefore $\zeta_{\mu}=0$. We deduce that $\eta_{\mu}$ vanishes outside $\Sigma$ and then on the whole domain $\Omega$. As a result, we obtain that $\mu=0$ and hence, $\mathcal{N}\left(\left(\mathcal{H}_{\Sigma}\right)^{*}\right)=\{0\}$. This implies that $\mathcal{R}\left(\mathcal{H}_{\Sigma}\right)$ is dense in $H^{1 / 2}(\Sigma)$. Since it is closed, $\mathcal{H}_{\Sigma}$ is onto and is then one-to-one. The proof is completed by the closed range theorem.

A dual variational formulation allows to give a mathematical sense to the corresponding $\chi_{\lambda} \in H^{1 / 2}\left(\omega_{\gamma}\right)$ for $\lambda \in L^{2}(\Sigma)$ and thereby to extend the operator $\mathcal{B}_{\Sigma}$ to $L^{2}(\Sigma)$. Here again, the elliptic theory tells that the resulting operator, still denoted by $\mathcal{B}_{\Sigma}$, is compact. Its adjoint is the restriction of $\left(\mathcal{B}_{\Sigma}\right)^{*}$ to $L^{2}(\Sigma)$. The following can be established in the same lines as Lemma 10.1.

Lemma 10.2. The linear operator $\mathcal{H}_{\Sigma}=\left(\mathcal{I}+\mathcal{B}_{\Sigma}\right)$ is an isomorphism on $L^{2}(\Sigma)$.

Acknowledgements. The authors are greatly thankful to anonymous reviewers for their comments which substantially improved the readability of the paper. This work was partially supported by the Ministère de l'Enseignement Supérieur, de la Recherche Scientifique et de la Technologie (TUNISIA) under the LR99ES-20 program and the "Coopération avec les Chercheurs Tunisiens Résidents à l'Étranger" program.

\section{REFERENCES}

[1] R.A. Adams, Sobolev Spaces. Academic Press, New York (1975).

[2] J.B. Apoung-Kamga and O. Pironneau, Numerical zoom for multiscale problems with an application to nuclear waste disposal. J. Comput. Phys. 224 (2007) 403-413.

[3] F. Ben Belgacem, M. Fournié, N. Gmati, F. Jelassi, Handling boundary conditions at infinity for some exterior problems by the alternating Schwarz method. C. R. Acad. Sci., Sér. 1 Math. 336 (2003) 277-282.

[4] F. Ben Belgacem, M. Fournié, N. Gmati and F. Jelassi, On the Schwarz algorithms for the elliptic exterior boundary value problems. ESAIM: M2AN 39 (2005) 693-714.

[5] C. Bernardi, Y. Maday and A.T. Patera, A New Non Conforming Approach to Domain Decomposition: The Mortar Element Method, in Non-linear Partial Differential Equations and their Applications 11, H. Brezis and J.-L. Lions Eds., Pitman/Wiley, London/New York (1994) 13-51.

[6] S. Bertoluzza, M. Ismaïl and B. Maury, The Fat Boundary Method: Semi-Discrete Scheme and Some Numerical experiments, in Domain decomposition methods in science and engineering, Lect. Notes Comput. Sci. Eng. 40, Springer, Berlin (2005) 513-520.

[7] F. Brezzi, J.L. Lions and O. Pironneau, On the chimera method. C. R. Acad. Sci., Sér. 1 Math. 332 (2001) 655-660.

[8] H.D. Bui, Fracture Mechanics: Inverse Problems and Solutions, Solid Mechanics and Its Applications 139. Springer (2006).

[9] P.-G. Ciarlet, The Finite Element Method for Elliptic Problems, Studies in Mathematics and Its Applications 4. North Holland (1978).

[10] D. Colton and R. Kress, Inverse acoustic and electromagnetic scattering theory, Applied Mathematical Sciences 93. Springer (1992).

[11] R. Dautray and J.-L. Lions, Analyse mathématique et calcul numérique pour les sciences et les techniques. Second edition, Masson, Paris (1988).

[12] G. Dolzmann and S. Müller, Estimates for Green's matrices of elliptic systems by $L^{p}$ theory. Manuscripta Math. 88 (1995) 261-273. 
[13] V. Frayssé, L. Giraud, G. Gratton and J. Langou, A Set of GMRES Routines for Real and Complex Arithmeticcs on High Performance Computers. CERFACS Technical Report TR/PA/03/3 (2003).

[14] R. Glowinski, J. He, J. Rappaz and J. Wagner, Approximation of multi-scale elliptic problems using patches of finite elements. C. R. Acad. Sci., Sér. 1 Math. 337 (2003) 679-684.

[15] R. Glowinski, J. He, J. Rappaz and J. Wagner, A multi-domain method for solving numerically multi-scale elliptic problems. C.R., Math. 338 (2004) 741-746.

[16] N. Gmati and B. Philippe, Comments on the GMRES convergence for preconditioned systems, in 6th International Conference on Large-Scale Scientific Computations, June 5-9, 2007, I. Lirkov, S. Margenov and J. Waśniewski Eds., Lect. Notes Comput. Sci. 4818, Springer-Verlag (2008) 40-51.

[17] P. Grisvard, Boundary value problems in non-smooth domains, Monographs and Studies in Mathematics 24. Pitman, London (1985).

[18] M. Grüter and K.-O Widman, The Green function for uniformly elliptic equations. Manuscripta Math. 37 (1982) 303-342.

[19] J. He, A. Lozinski and J. Rappaz, Accelerating the method of finite element patches using approximately harmonic functions. C. R. Acad. Sci., Sér. 1 Math. 345 (2007) 107-112.

[20] F. Hecht, EMC2, Éditeur de Maillage et de Contours en 2 Dimensions. http://www-rocq1.inria.fr/gamma/cdrom/www/emc2.

[21] F. Hecht, A. Lozinski and O. Pironneau, Numerical Zoom and the Schwarz Algorithm, in Domain Decomposition Methods in Science and Engineering XVIII, Lecture Notes in Computational Science and Engineering 70, M. Bercovier, M.J. Gander, R. Kornhuber and O. Widlund Eds., Springer (2008).

[22] M. Ismaïl, The Fat Boundary Method for the Numerical Resolution of Elliptic Problems in Perforated Domains. Application to $3 D$ Fluid Flows. Ph.D. thesis, Université UPMC, Paris VI, France (2004).

[23] F. Jelassi, Sur les méthodes de Schwarz pour les problèmes extérieurs. Application au calcul des courants de Foucault en électrotechnique. Ph.D. Thesis, Université Paul Sabatier, Toulouse III, France (2006).

[24] P.-L. Lions, On the alternating Schwarz method I., in First International Symposium on Domain Decomposition Methods for Partial Differential Equations, R. Gowinski, G.H. Golub, G.A. Meurant and J. Périaux Eds., SIAM, Philadelphia (1988) 1-42.

[25] J. Liu and J.M. Jin, A novel hybridization of higher order finite element and boundary integral methods for electromagnetic scattering and radiation problems. IEEE Trans. Antennas Propag. 49 (2001) 1794-1806.

[26] B. Lucquin and O. Pironneau, Introduction to Scientific Computing. John Wiley \& Sons Ltd., Inc., New York (1998).

[27] D. Martin, MELINA, Guide de l'utilisateur. I.R.M.A.R., Université de Rennes I/E.N.S.T.A. Paris, France (2000). http://perso.univ-rennes1.fr/daniel.martin/melina.

[28] B. Maury, A fat boundary method for the Poisson equation in a domain with holes. J. Sci. Comp. 16 (2001) 319-339.

[29] I. Moret, A note on the superlinear convergence of GMRES. SIAM J. Numer. Anal. 34 (1997) 513-516.

[30] J.C. Nédélec, Acoustic and Electromagnetic Equations. Integral Representations for Harmonic Problems. Springer (2000).

[31] O. Pironneau, Numerical Zoom for Localized Multi-Scale Problems. Invited conference, MAFELAP, Brunel University, London (2009).

[32] A. Quarteroni and A. Valli, Domain Decomposition Methods for Partial Differential Equations. Oxford Science Publications (1999).

[33] A. Quarteroni, A Veneziani and P. Zunino, A domain decomposition method for advection-diffusion processes with application to blood solutes. SIAM J. Sci. Comput. 23 (2002) 1959-1980.

[34] Y. Saad, Iterative methods for sparse linear systems. Second edition, SIAM (2003).

[35] R. Schinzinger and P.A.A. Laura, Conformal Mapping: Methods and Applications. Amsterdam: Elsevier Science Publishers (1991).

[36] A. Toselli and O.B. Widlund, Domain decomposition methods-algorithms and theory, Springer Series in Computational Mathematics 34. Springer-Verlag, Berlin (2005).

[37] H. Weyl, Das asymptotische Verteilungsgesetz der Eigenwerte linearer partieller Differentialgleichungen (mit einer Anwendung auf die Theorie der Hohlraumstrahlung). Math. Ann. 71 (1912) 441-479. 